\title{
Attività sismica in Italia nell'anno 1970
}

\author{
(Seismic activity in Italy during the year 1970)
}

\author{
C. GaspakiN1 - I. Giovaxl (*)
}

Riceruto il 2 Aprile: 1973

Ressusto. - Somo atalti riportati in catalogo i terremoti avenuli in Italia nel 1970. I dati ad essi relativi sono stati completati con un graslo di attendlibilità, riferifo alle coortiuate epicentrali, alla profouclita e alla marnitudo. Her il ealeolu della magnitulu, non essendo questo date sempre ottenibile strumentaluente, sono stati atoperati i parametri di correlazione con lintensita per i relativi distretti sismotettonici. Fell ultima parte sono deseritti quei terremoti per i quali è stato possibile eflettuare un esauc det tagrlialu.

Summars. - Eathquakes oceured in ltaly during 1970 have been reported in the list. Their relative dates have been completed with acenracy, related to the coordinated fpicentres, the depth and the magnitude. For the caleulatim of the matuitule, parameters of correlation with the intensity for the relative seismo-tectonic langers were used, as these data is not always olbtained instrumentally. The eartheluke for which it was possible in effectuate a detailed examination are described in the last part.

\section{§ 1 - CONSIDFRAZIONI F DESCRIZIONE DELL'ELENCO}

Si sono riportati in ortine cronologieo tre gruppi ai sismi, le cui caratiteristiche sono:

- 10 gruppo - Comprende tutti quei sismi li cui si avevano sufticienti notizie da poterli lowtare llettagliatamente (terza parte dell'indaginte).

(*) Osgervatorio Sismicen di Mnnte Porzio (Roma). Istituto Nazimale di Geofisien. 
- 20 gruppo - Sismi le eni determinazioni ipocentrali sono state effetfute da stazioni estere in base ai tati ta esse alaborati, e per $\mathrm{i}$ quali le nostre stazioni non hanno formito tati sufficienti alla determinazione al individuazione degli eventi stessi.

- 30 gruppo - Terremoti di cui si avevano le sole notizie macrosismiche e qualche registrazione totalmente insufficiente al una ricerca aceurata.

JNTENSIT MACRosIsma - E sempre riportata in base alla scala Mercalli-Sieberg. Tel catalogo vi sono due colonne inticate con $I$ e $I_{o}$. La $I$ si riferisee all'intensiti tlel lnogo maggiormente colpito, ma non necessariamente al valore che essa tassumerebbe all'epicentro. L'intensiti macrosismica all'epicentro, invece is inticata con $I_{o}$.

Le fonti a cni abbiamo attinto sono: l'Ufficio Centrale di Meteorologia e Geodinamica (sez. sismiea), la "Stampa " locale e nazionale, l'acquisizione diretta.

Maritudno - La magnitudo is stata, quando possibile, ricavata dai sismogrammi $(M)$, altrimenti è macrosismica ( $\left.M^{*}\right)$.

Coomplyate ficentrali - In relazione al tipo di notizie in nostro possesso, sono stati nsati altemativamente per il caleolo dei dati ipocentrali, i metodi Takth tusi (19), Caloi (1), Caloi-Peronaci ( $)$ ).

Le coordinate sono date a seconda del valore di approssimazione, in gradi e tlecimi o centesimi.

Propoxorri - È stata ricavata mediante il metodo di Caloi $(3,4)$, altrimenti è determinata dalla macrosismica.

§ 2 - ATTFNDIBILTA DEI DATI RIPORTATI NFLL'ELENCo.

In questo lavoro, si è inteso dare un valore di attendibilitì ai dati riportati nell'elenco, onde evitare confusioni sulla valutazione dei dati stessi.

Il valore di attendibilità è costituito ta tre cifre: la prima si riferisce alle coordinate epicentrali, la seconda si riferisce alla profonditì ipocentrale, la terza si riferisce all magnitudo. 


\begin{tabular}{|c|c|c|c|c|c|c|c|c|c|c|c|c|c|c|c|c|c|}
\hline \multirow{2}{*}{ N. } & \multirow{2}{*}{\multicolumn{2}{|c|}{ 1)ata }} & \multicolumn{3}{|c|}{ T.MI.G. } & \multicolumn{2}{|l|}{ RE G I ONE } & EPICENTRO MACROS & Isuico & Coorl. & picent. & & & & & & \\
\hline & & & $h m$ & $m s$ & $s$ & Zona & J)is. & Jocalità & $I$ & $\varphi^{\circ}$ & $\hat{\lambda}^{\circ}$ & & & & & & \\
\hline 1 & 3 & Gennaio & $23+$ & 44 & 20 & Lago di Garla & 4 & Gavarclo & IV & 45,6 & 10,6 & B & 3,8 & & IV & 443 & $\begin{array}{l}\text { Replica alle } 24^{\mathrm{n}}+0^{\mathrm{m}} \text { cil alle } 12^{\mathrm{n}} 25^{\mathrm{m}} \\
\text { clel giorno suceessivo - (v. Fig. } 3 \text { ) }\end{array}$ \\
\hline $\begin{array}{l}2 \\
3\end{array}$ & $\begin{array}{l}21 \\
24\end{array}$ & $\begin{array}{l}\text { Gennaio } \\
\text { Gennaio }\end{array}$ & $\begin{array}{ll}18 & 3 \\
09 & 3\end{array}$ & $\begin{array}{l}36 \\
30\end{array}$ & 25 & $\begin{array}{l}\text { Gargano } \\
\text { Gargano }\end{array}$ & $\begin{array}{l}11 \\
11\end{array}$ & $\begin{array}{l}\text { Vico Garganiro } \\
\text { Cagnano Varano }\end{array}$ & $\begin{array}{l}\text { VI } \\
\text { IV }\end{array}$ & $\begin{array}{l}41,9 \\
41,8\end{array}$ & $\begin{array}{l}16,4 \\
15,8\end{array}$ & $\begin{array}{l}\mathrm{B} \\
\mathrm{B}\end{array}$ & $\begin{array}{l}4,0 \\
2,7\end{array}$ & & $\begin{array}{l}\text { VI } \\
\text { IV }\end{array}$ & $\begin{array}{l}443 \\
4+3\end{array}$ & Il 23.1 dehole repliea - (v. Fig. 4 ) \\
\hline 4 & 29 & Gennaio & $\begin{cases}11 & 0 \\
11 & 0\end{cases}$ & $\begin{array}{l}09 \\
69\end{array}$ & $\frac{24,1}{24}$ & Basso Tirreno & 16 & & & $\begin{array}{l}+1,0 \\
38,8 \\
38,7\end{array}$ & $\begin{array}{l}10,8 \\
14,9 \\
14,8\end{array}$ & 280 & & 4,7 & & $\begin{array}{l}+43 \\
221 \\
22-\end{array}$ & $\begin{array}{l}\text { U.S.C.G.S. } \\
\text { B.C.I.S. }\end{array}$ \\
\hline 5 & 9 & Fehbraio & 073 & 39 & 08 & Appennino Forlivese & 7 & Galeata & VI & 43,97 & 12,10 & 28 & & 4,5 & vi & 221 & seguono 2 repliche $(* *)$ \\
\hline 6) & 13 & Fcbhraio & 210 & 08 & 34 & il. Peloritani & 13 & Venetico & $\mathrm{V}$ & 38,2 & 15,4 & A & 2,9 & & $\mathrm{v}$ & $4+3$ & \\
\hline 7 & 17 & Febhraio & 073 & 32 & 04,9 & Valle clel Crati & 13 & & & 39,7 & 16,1 & 262 & & 4,4 & & 221 & U.S.C.G.S. \\
\hline 8 & 22 & Febbraio & 113 & 30 & & P'rov, di Trapani & 15 & Calatafiui & II-III & 37,9 & 12,7 & B & 2,6 & & & 443 & ur \\
\hline 9 & 26 & Fehbrain & 095 & 58 & & l'rov, lli Trajani & 15 & Calatafimi & II-III & 37,9 & 12,7 & B & 2,6 & & & 443 & nr \\
\hline 10 & 28 & Fehbraio & & & & Campi Flenrei & 9 & Pozzuoli & & - & - & & & & & & Fenomeno microsismico \\
\hline 11 & 9 & Marzo & 205 & 59 & 31 & Etna & & & IV.V & 37,8 & 15,2 & $\Lambda$ & 2,6 & & & 443 & $\begin{array}{l}\text { Seguono } 17 \text { repliclic tra il II o } \\
\text { III gruto }\end{array}$ \\
\hline 12 & 10 & Marzo & 140 & 00 & & Lago di Garda & 5 & Cavedine & III-IV & 46,0 & 11,0 & B & 3,1 & & & 443 & $\mathrm{nr}$ \\
\hline 13 & 13 & Marzo & 063 & 30 & & Ріпла di Silyari & 13 & Alto Monte & II-III & 39,7 & 16,1 & $\Lambda$ & 2,0 & & & 443 & $\mathrm{mr}$ \\
\hline 14 & 23 & Marzo & $12+$ & 43 & 24 & Prov. di Terni & 8 & Terni & V & 42,5 & 12,6 & $\mathrm{~B}$ & 3,2 & & $v$ & 443 & \\
\hline 15 & 5 & Aprile & 185 & 53 & 32 & Valle Maira & 2 & Castcl Magro & IV.V & $4+, 5$ & 7,2 & $\geqslant 10$ & 3,5 & & IV.Y & $3+3$ & (v. Fig. 5) \\
\hline 16 & 17 & Aprile & 093 & 37 & 49 & l'rov. di Frosinone & 9 & Cassino & v & $41, \tilde{5}$ & 13,8 & $\mathrm{~B}$ & 3,3 & & & $4+3$ & \\
\hline 17 & 19 & Aprile & 181 & 16 & 32 & Lago di Garila & 4 & Gavardo & VI & 45,65 & 10,47 & 13 & & 4,3 & VI & 111 & $(* *)$ \\
\hline $\begin{array}{l}18 \\
19\end{array}$ & $\begin{array}{l}21 \\
26\end{array}$ & $\begin{array}{l}\text { Aprile } \\
\text { Aprile }\end{array}$ & $\begin{array}{ll}00 & 1 \\
19 & 1\end{array}$ & $\begin{array}{l}10 \\
14\end{array}$ & $\begin{array}{l}17 \\
40\end{array}$ & $\begin{array}{l}\text { Prov. Ii Ricti } \\
\text { Golfo di Taranto }\end{array}$ & 8 & Canneto & III & $\begin{array}{l}42,2 \\
30.8\end{array}$ & 12,7 & B & 2,5 & & & 443 & \\
\hline $\begin{array}{l}19 \\
20\end{array}$ & $\begin{array}{r}26 \\
3\end{array}$ & $\begin{array}{l}\text { Aprile } \\
\text { Mlaggio }\end{array}$ & 041 & 17 & 41 & Prov. di Parma & 6 & & & $\begin{array}{l}39,8 \\
44,9\end{array}$ & $\begin{array}{l}17,8 \\
10,4\end{array}$ & & & 4,3 & & $\begin{array}{l}2-1 \\
2--\end{array}$ & $\begin{array}{l}\text { Epicentro in mare - ATII } \\
\text { B.C.I.S. }\end{array}$ \\
\hline 21 & 5 & Maggio & $12+$ & 49 & 56 & Prov. di Parma & 7 & & & 44,8 & 10,2 & 0 & & & & $22-$ & B.C.I.S. \\
\hline 22 & 6 & Maggio & 091 & 16 & 58 & P'rov. di Savona & 1 & Vendone & $\mathrm{V}$ & 44,0 & 8,1 & B & 3,7 & & $\mathrm{v}$ & 343 & (v. Fig. 6) \\
\hline 23 & 8 & Maggio & 052 & 20 & & Valle tlel Crati & 13 & Cerzets & V & 39,5 & 16,1 & B & 3,6 & & v & 443 & $\mathrm{nr}$ \\
\hline 24 & 17 & Maggio & 053 & 30 & & Trapanese & 14 & l'artanna & IV & 37,7 & 12,9 & B & 2,9 & & & $4+3$ & $\mathrm{nr}$ \\
\hline 25 & 25 & II:uggio & 125 & 55 & 24 & Prov. di Terui & 8 & Terni & V.VI & 42,6 & 12,5 & & & $3,5(*)$ & V.VI & $2-1$ & B.C.I.S. $-\left(^{*}\right)$ RMEP \\
\hline 26 & 26 & Maggio & 120 & 04 & 34 & Prov. di Terni & 8 & Terni & $v$ & $+2,6$ & 12,5 & & & & $\mathrm{v}$ & $2--$ & Repliea del precedente - B.C.I.S. \\
\hline 27 & 5 & Giugno & 092 & 20) & 55,6 & Basso Tirreno & 16 & & & 39,2 & 15,4 & 262 & & 4,4 & & 221 & $\begin{array}{l}\text { Avvertito in Prov. di Reggio Ca- } \\
\text { lahria - U.S.C.G.S. }\end{array}$ \\
\hline 28 & 14 & Gimgno & 115 & 57 & 44 & Padlana Veronese & 6 & & & $4 \overline{5}, 3$ & 11,2 & & & & & $2-3$ & B.C.I.S. \\
\hline 29 & 16 & Gingno & $04 \quad 1$ & 19 & 26 & Prov. di Parma & 6 & & & 44,8 & 10,4 & & & & & $2-3$ & $\begin{array}{l}5 \text { repliche tra le } 0^{04^{\mathrm{n}} 20^{\mathrm{m}}} \text { e le } \\
06^{\mathrm{n}} 30^{\mathrm{m}} 1^{\mathrm{J} J} \mathrm{~J}-1 \text {.C.I.S. }\end{array}$ \\
\hline 30 & 18 & Gingno & 090 & 03 & 03 & Nurra & 17 & & & 41,0 & 07,8 & $\geqslant 15$ & & & & $24-$ & $\mathrm{E}_{\mathrm{p}}$ icentro in mare - B.C.I.S. \\
\hline 31 & 18 & Gingno & 103 & 341 & (18) & P'rov. di Imperia & 1 & Vallecrosia & IV & 43,8 & 7,6 & B & 2,9 & & IV & 443 & \\
\hline 32 & 20 & Gingno & & & & Prov. di Macerata & 10 & Macerata & III & 43,3 & 13,5 & $\geqslant 10$ & 2,3 & & & $4+3$ & $\mathrm{nr}$ \\
\hline 33 & 29 & Gingno & 125 & 54 & 13 & Prov. di Catanzaro & 13 & Catanzaro & III & 38,9 & 16,6 & $\geqslant 10$ & 2,7 & & III & 443 & \\
\hline 34 & 29 & Giugno & $1+2$ & 22 & 52 & Piana di Catanzaro & 13 & Pentone & IV & 38,9 & 16,6 & $\geqslant 10$ & 3,2 & & IV & 242 & $(* *)$ \\
\hline 35 & G & Luglio & 220 & 06 & 41 & Valle del Toppino & 8 & Folizno & II-III & 42,9 & 12,7 & B & 2,3 & & II-III & 443 & \\
\hline 36 & 7 & Luglio & 060 & 04 & 24 & Val Nerina & 8 & S. Anatolia di Nareo & v & 42,78 & 12,88 & 12 & & 4,0 & V & 231 & $(* *)$ \\
\hline $\begin{array}{l}37 \\
38\end{array}$ & 16 & Luglio & 135 & 52 & 21 & Prov, di Campohasso & $\begin{array}{l}8 \\
8\end{array}$ & $\begin{array}{l}\text { Boiano } \\
\text { Arrone }\end{array}$ & $\begin{array}{l}\text { III } \\
\text { II }\end{array}$ & $\begin{array}{l}41,5 \\
42,6\end{array}$ & 14,5 & $\mathrm{~B}$ & $\begin{array}{l}2,2 \\
2,1\end{array}$ & & & 443 & \\
\hline $\begin{array}{l}38 \\
39\end{array}$ & $\begin{array}{l}16 \\
30\end{array}$ & $\begin{array}{l}\text { 1uglio } \\
\text { Lnglin }\end{array}$ & 22 & 20 & $\begin{array}{l}+3 \\
03\end{array}$ & $\begin{array}{l}\text { Val Nerina } \\
\text { Prov. Ili Caserta }\end{array}$ & $\begin{array}{l}8 \\
9\end{array}$ & $\begin{array}{l}\text { Arrone } \\
\text { Apice }\end{array}$ & $\mathrm{v}$ & 4 & $\begin{array}{l}12,8 \\
14,9\end{array}$ & $\mathrm{~B}$ & 3,1 & & $\mathrm{~V}$ & $\begin{array}{l}423 \\
4+3\end{array}$ & Repliea alle $22^{\mathrm{n}} 26^{\mathrm{m}} 30^{\mathrm{s}}-(\mathrm{v}$. Fig. 7$\}$ \\
\hline 40 & 8 & Agosto & $104 \frac{2}{2}$ & 27 & $\begin{array}{l}17,4 \\
22\end{array}$ & Alto Adriatice & 6 & & & $\begin{array}{l}44,7 \\
44,2\end{array}$ & $\begin{array}{l}12,8 \\
12,9\end{array}$ & 27 & & $4,2(*)$ & & 221 & $\begin{array}{l}\text { U.S.C.G.S. }-\left(^{*}\right) \text { R.IP } \\
\text { B.C.I.S. }\end{array}$ \\
\hline 41 & 16 & Agnsto & 1104 & $\begin{array}{l}45 \\
45\end{array}$ & $\begin{array}{l}21 \\
25\end{array}$ & Mar Jonio & 13 & Catanzaro & IV & $\begin{array}{l}37,9 \\
38,0\end{array}$ & $\begin{array}{l}16,5 \\
16,9\end{array}$ & 52 & & $\begin{array}{l}4,3 \\
5,0(*)\end{array}$ & & $\begin{array}{l}221 \\
221\end{array}$ & $\begin{array}{l}\text { U.S.C.G.S. Epirentro in mare } \\
\text { 1.C.I.S. }\left(^{*}\right) \text { ATH }\end{array}$ \\
\hline 42 & 18 & Agosto & $18+$ & 42 & 45 & Vall ili Cliana & 7 & & & 43,3 & 11,6 & & & & & $2--$ & B.C.I.S. \\
\hline 43 & 19 & Agosto & 121 & 19 & 46 & Colline Metallifere & 7 & Menterotondo Maritt. & VI & 43,09 & 10,77 & 10 & & $4,4(*)$ & VI & 121 & $\left(^{* *}\right)-\left(^{*}\right)$ RMIP \\
\hline 44 & 20 & Agosto & 02 & 03 & & Penis. Salentina & 12 & Otranto & $\mathrm{V}$ & 40,2 & 18,5 & B & 3,5 & & $\mathrm{v}$ & 443 & $n r-($ v. Fig. 8$)$ \\
\hline 45 & 20 & Agosto & 123 & 30 & & Colline Matallifere & 7 & Suvereto & IV-Y & 43,1 & 10,7 & $\geqslant 10$ & 2,8 & & IV.Y & 443 & $\mathrm{nr}$ \\
\hline 46 & 20 & Agrosto & 190 & 00) & & Gargatno & 11 & Iscluitella & IV & 41,9 & 15,9 & $\geqslant 10$ & 2,7 & & IV & 443 & nr \\
\hline 47 & 28 & Agosto & 205 & 50) & 59 & Apl. Modenese & 7 & & & 44,6 & 10,9 & 0 & & & & $22-$ & B.C.I.S. \\
\hline 48 & 7 & Scttemlure & 140 & 02 & 21 & App. Timbro & 8 & Sellano & VI & 42,87 & 12,95 & 16 & & $4,1\left(^{*}\right)$ & VI & 121 & $\left({ }^{*}\right) \mathrm{RMIP}-\left({ }^{* *}\right)$ \\
\hline 49 & 12 & Settemlre & 140 & 00 & & P'rov, ili Macerata & 10 & Rerantati & IV & 43,4 & 13,5 & $\geqslant 10$ & 3,0 & & IV & 443 & ir - Premonitrice di II alle $08^{14} 00^{\mathrm{m}}$ \\
\hline 50 & 26 & Settemlure & $16+$ & +2 & 28 & Forlivese & 6 & Cesena & V.VI & 44,1 & 12,3 & & & $4,4(*)$ & V.VI & $2-1$ & B.C.I.S. $-\left({ }^{*}\right)$ RMP \\
\hline 51 & 27 & Settembre & 191 & 18 & 30 & Prov. di C'asertit & 9 & Mignano Monte Lungo & VII & 41,37 & 14,02 & 7 & & $3,9(*)$ & VII & 111 & $(* *)-(*)$ RMI \\
\hline 52 & 28 & Settembre & 153 & 34 & 31 & Colli Albani & 9 & Colonia & III & 41,7 & 12,7 & $\leqslant 10$ & 2,2 & & & 443 & \\
\hline 53 & 29 & Settemlre & 000 & 06 & 17 & Colli Allaai & 9 & Colonna & $\mathrm{V}$ & 41,7 & 12,7 & $1-5$ & & $3,4(*)$ & V & 211 & $\left(^{*}\right) \mathrm{RMP}-\left({ }^{* *}\right)$ \\
\hline 54 & 6 & Ottobre & 065 & 51 & & Prov. di Caserta & 9 & Mignano Monte Lungo & $\mathrm{V}$ & 41,4 & 14,0 & 7 & 3,0 & & $\mathrm{v}$ & 212 & \\
\hline 55 & 7 & Ottohre & 000 & 05 & 09 & Mindonie & 14 & Caltavulturo & $v$ & 37,8 & 13,9 & $\mathrm{~B}$ & 3,3 & & $r$ & $4+3$ & Replica alle $02^{\mathrm{h}} 10^{\mathrm{m}}$ \\
\hline 56 & 10 & Ottohre & 050 & 00 & & Prov. di Cuneo & 2 & Roaschia & IV & 44,5 & 7,5 & $\geqslant 15$ & 3,0 & & & $4+3$ & $\mathrm{nr}$ - replica di IV alle $22^{\mathrm{n}} 15^{\mathrm{m}}$ \\
\hline 57 & 18 & ottobre & 034 & 46 & & Colli Albani & 9 & Laglietto & III-IV & 41,7 & 12,7 & $\geqslant 10$ & 3,0 & & & 443 & \\
\hline 58 & 20 & Ottobre & 054 & 45 & & Prov. di Cuneo & 2 & Valdieri & IV & 44,4 & 7,5 & $\geqslant 10$ & 3,0 & & & $4+3$ & $\mathrm{nr}$ - replica alle $04^{\mathrm{h} 25^{\mathrm{m}}}$ il $23 . \mathrm{X}$. \\
\hline 59 & 22 & Ottobre & 035 & 50 & & Prov. di Potenza & 8 & Castelgrande & $\mathrm{V}$ & 40,8 & 15,4 & 13 & 3,1 & & $\mathrm{v}$ & 443 & 2 repliche - $\mathrm{nr}$ \\
\hline 60 & 26 & Ottohre & 153 & 35 & & Valle del Belice & 15 & Partanna & III & 37,7 & 12,9 & B & 2,6 & & & 443 & ur \\
\hline 61 & 31 & Ottohre & 011 & 16 & 20 & Prov. di Caserta & 9 & Roccamonfina & V.YI & 41,4 & 13,8 & 56 & 3,4 & & V.VI & 212 & \\
\hline 62 & 2 & Sovemilre & 032 & 24 & 51 & Alta Val Tiberina & 7 & San Sepolero & IV & 43,7 & 12,0 & B & 3,5 & & IV & 242 & I.N.G. - (v. Fig. 9) \\
\hline 63 & 2 & Xovembre & $08+$ & 42 & 12 & l'rov. di Verona & 6 & & & 45,1 & 11,1 & & & & & $2--$ & B.C.I.S. \\
\hline 64 & 2 & Novembre & 144 & 46 & II & Prov. „li Caserta & 9 & Mignano Monte Linngo & II-III & 41,4 & 14,0 & 5 & 2,1 & & & 443 & \\
\hline 65 & 19 & Novembre & 181 & 12 & 02 & Frign:mo & $7 \mathrm{~b}$ & & & 44,6 & 10,6 & & & & & $"--$ & B.C.I.S. \\
\hline 66 & 21 & Novembre & 073 & 36 & +6 & Prov. Ili Casert: & 9 & Mignano Monte Lungo & V.VI & 41,4 & $14,1)$ & 6 & 3,4 & & V.YI & 212 & \\
\hline 67 & 22 & Novembre & $23+5-r a r-3$ & 47 & 15 & Prov. di Casertat & 9 & s. Vittore & $\mathrm{VI}$ & 41,4 & 14,0 & 6 & 3,5 & & VI & 212 & (v. Fig. 10) \\
\hline 68 & 26 & Novembre & 035 & 57 & 19 & Prov. di Caserta & 9 & S. Pietro Infine & v & 41,4 & 14,0 & 6 & 3,2 & & v & 212 & \\
\hline 69 & 18 & Jicembre & 052 & 25 & 50 & Colli Alhani & 9 & Laglictto & III & 41,7 & 12,7 & $A$ & 2,2 & & & 443 & \\
\hline 70 & 25 & Jicembre & 021 & 14 & & Prov. di Caserta & 9 & Mignano Nonte Lungo & $\mathrm{V}$ & 41,4 & 14,0 & $5-6$ & 3,2 & & $r$ & 212 & \\
\hline 71 & 30 & IDicentbre & 022 & 20 & 05 & Ligurial Occiclentales & 1 & Calice ligure & $\mathrm{V}$ & 44,2 & 8,3 & B & 3,8 & & $v$ & 443 & \\
\hline 72 & 30 & Dicembre & 051 & 16 & & Valle del Belice & 15 & Partanna & $V \cdot V I$ & 37,7 & 12,9 & B & 3,6 & & V.VI & 443 & $\mathrm{nr}$ \\
\hline 73 & 31 & Dicembre & 221 & 04 & 46 & Liguria Oecidentale & 1 & P'ietrat Ligure & VI & 44,15 & 8,28 & 9 & & 4,3 & ri & 111 & $(* *)$ \\
\hline
\end{tabular}

(2) I dati riportati, si riferisenno per ciascun rigo, a quelli forniti dall'lnte inclicato, in nota. I clati macrosismici sono quelli acquisiti dagli autori.

Je sigle corrispontono a:

I.N.G. = Istituto Nazionale li Genfisica

B.C.I.S. = Bureau Central International de la Seismologic - Strasbourg

Li.S.C.G.S. = Vinited States Coast And Feodetic Survey

RMP - Istituto Nazionale di Geofisica - Roma Monte Porzio

PID $\quad=$ Istituto Nazionale uli Gcofisica - Padova

$\mathrm{ATH}=$ National Observatory of Atlien

(**) = Facenti parte di quelli trattati nel paragrafo 4

$\mathrm{nr} \quad=$ Non si ì avuta nessuna registrazione tel sisma. 
Coordisate EPICExtrat - Sono slate fatte quatto classi con le seguenti caratteristiclus:

1) - Ricavate con metodo analitieo.

2) - Ricavate tramite metodo grafico.

3) - Rilerite all'epicentro macrosismico, se questo è stato inequivocabilmente determinato.

1) - Sempre riferite all'epicentro macrosismico ma con minore precisione del precedente dafa la scarsa densita di centri abitati nelle zone interessate dal sisma.

Profoxdit tPocextrale - E stata effettuata la seguente suddivisione:

1) - Metolo analitien.

2) - Hetodo grafico.

3) - Metodo macrosismico. Jat profomditì ì calcolata in base alle isosiste, e per terremoti avvenuti presumibilmente ma non al disotto delliv crosta terrestre, la relazione usata è

$$
h=\frac{r}{\sqrt[10^{I_{0}-I}-1]{3}-1}
$$

love $r$ è il ragroio medio dall'epicentro all'isosista di grado $I, x_{0}$ l'intensità macrosismica ilellepicentro.

Lat formula precelente non puo essere usata per $i$ sismi avvenuti in localila sopraggiacenti a potenti formazioni sedimentarie data l'esaltazione che la intensiti subisce in queste zone.

4) - Stimate in base alle caratteristiche sismotettoniche della regione. Lar profonditib è assegnata da conoseenze già acquisite, tenendo presente la tettonica di zona.

Rispetto alla profonditi i terremoti sono stati divisi in qualtro gruppi:
A) $-h \leqslant 5 \mathrm{~km}$
B) $-5 \mathrm{~km}<h \leqslant 25 \mathrm{~km}$
C) $-25 \mathrm{~km}<\pi \leqslant 60 \mathrm{~km}$
D) $-h>60 \mathrm{~km}$. 
MLAgritudo - Sono state fatte tre chassi:

1) - Ricavata analiticamente dal sismogramma.

2) - Macrosismica, con profondità nota.

3) - Macrosismica, con la prolondità stimata (vedi elasse 1 per Ia profondití).

\section{$\S 3$ - DistreTti sismoteTroNicI}

Nello stulio della sismicitì d'Italia, il territorio è stato suldiviso in distretti tettonici (fig. 1). Muggiori chiarimenti appariranno nella pubblicazione relativa al periodo sismico di Ancona. Appresso diamo m elenco dei distretti con le loro caratteristiche fondamentali.

Distretto 1 - Mle Manímune - Ricoprimenti pennidiei costituiti da scisti cristallini pretriassicti ricoperti da terreni mesozoici.

Distretto 2 - AIPI OCCIDENTaLI - Coltri inferiori (elvetidi), costituite dai nuclei cristallini ercinici e tettonieamente rinteressati dal corrugamento alpino, coltri sedimentarie delle elvetidi e dell'avampace.

Distreto 3 - ALPI SETLEXTRIONALI - eostituite tlai rieoprimenti Austroalpini superiori e dalle zone para-autoctone. Alpi calcarec settentrionaii.

Distretto $4-\bar{j}$ - ALPI XIFRIDIoriLI - Costibute prevalentemente dalle formazioni calearee, si hamlo anche manifestazioni eruttive erciniche. Tipica regione a pieglie prevalentemente vergenti a Sul, Il tistretto + i compreso tra il Lago di Como e la Valle Gindienue, il distretto 5 dalla. Falle Giudicarie alle Alpi Ininariele. Tale suddivisione ò stata eftettuata causa la maggiore complessita tettonica della zona orientale rispetto l'occilentale.

Distreto 6 - VALLe Pansxa - Costituita dai depositi fluvioglaciali e alluvionali ricoprenti lo strutture preplioceniche. In questo distretto si is anche posto il rilievo ilel INonferrato $(6 \mathrm{~A})$.

Distreto $z$ - APPENTo Ligure-Tosco-Romagolo - Costituito dalle serie alloctone e antoctome, dal massiceio di Voltri (7A), alalle falde liguridi (7B). Si hanno tue motivi tettomici fondamentali, quello delle strutture a ricoprimenti delle "Argille Seagliose " e quello doxuto a doformazioni del substrato rigido per faglio longitudinali. 


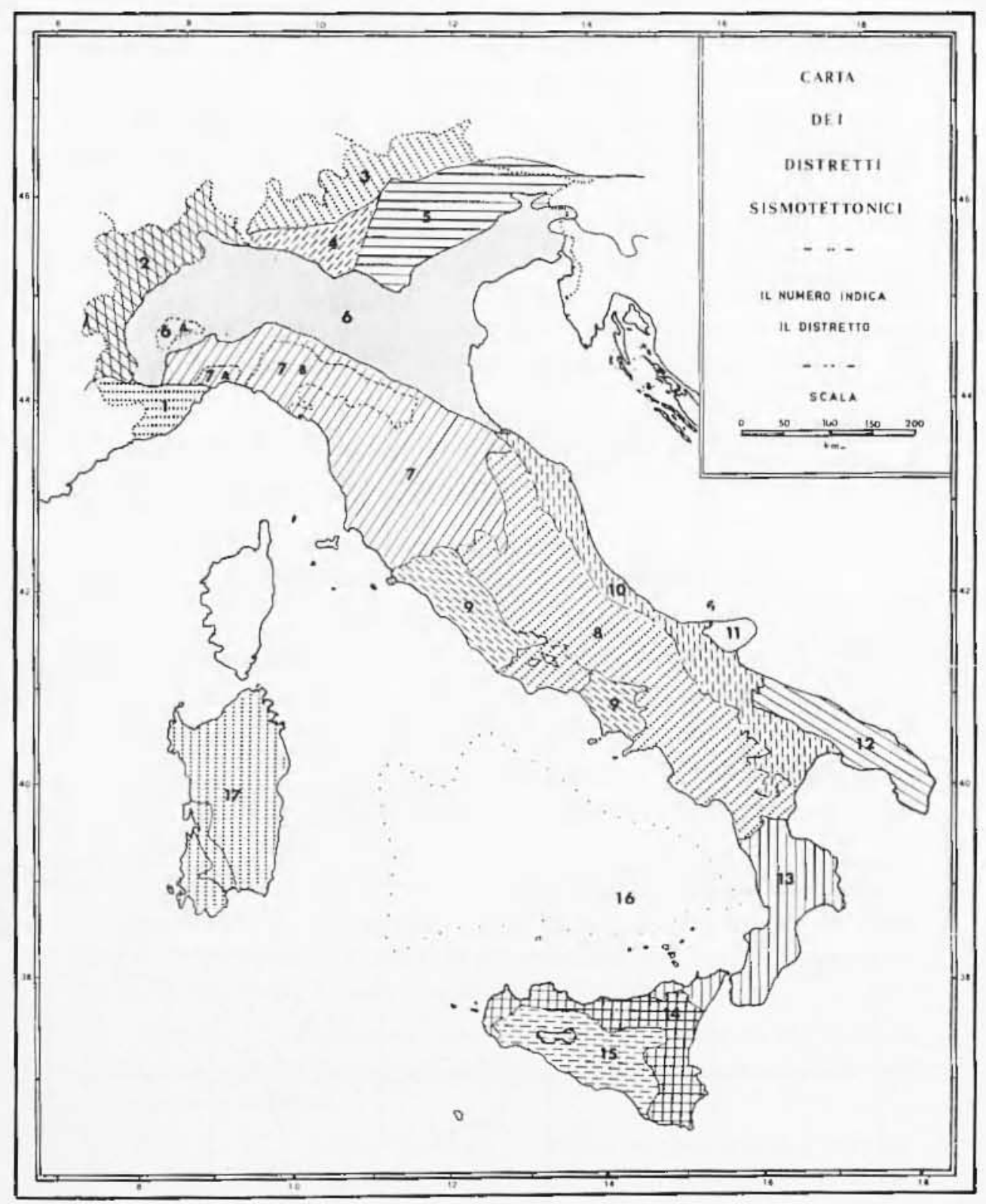

Fig. 1 


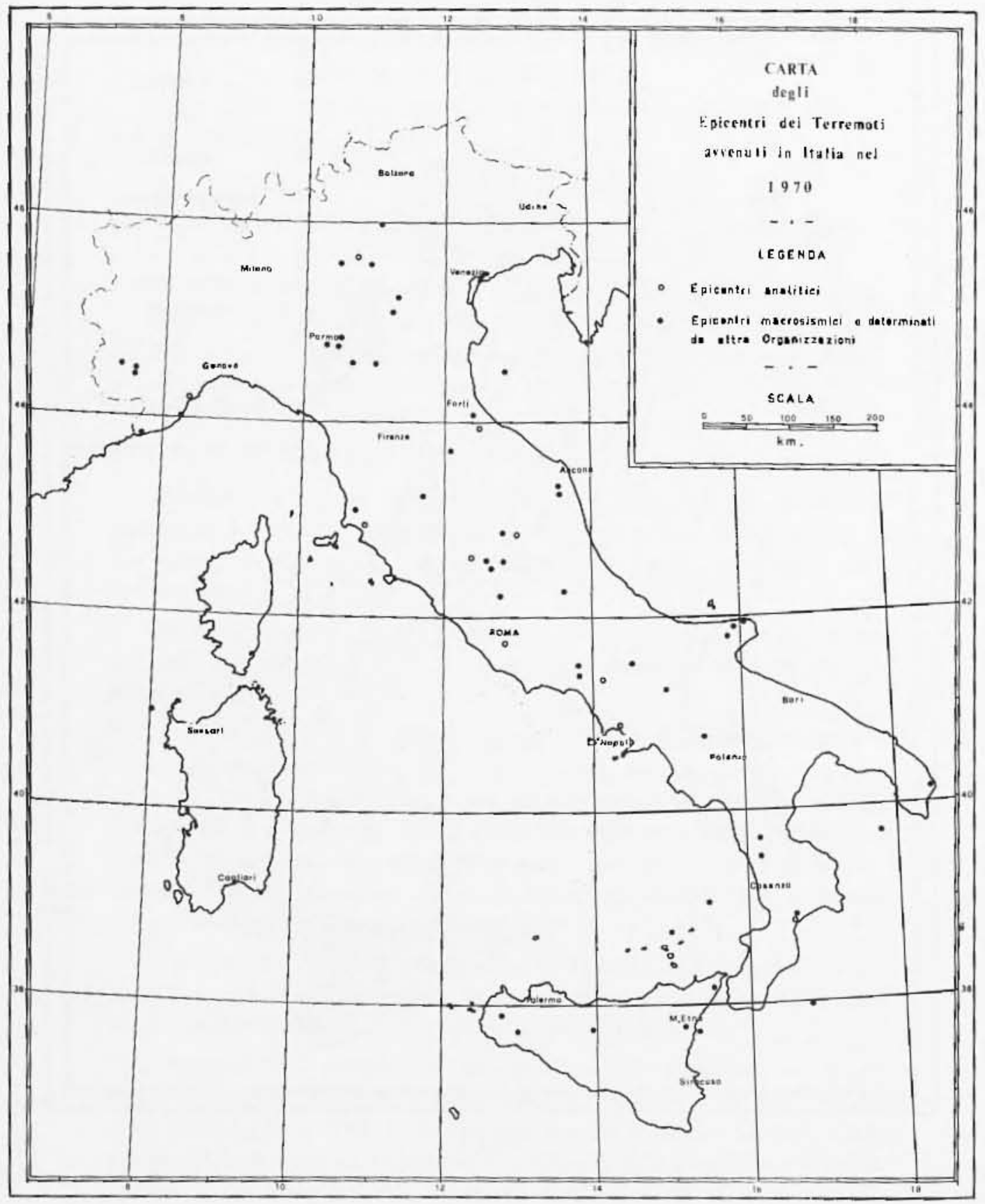

Fig. 2 
Distretto 8 - APIENXINo UMBRo-AIRGHGLXO-ABRUZZESE - Costituito essenzialmente da serie carbonaticle. Ia struttura è tatis da anticlinali e sinclinali regolari e sviluppate. Questo thst roto comprende a sud anche gli Aurunci, gli Ausoni, la Penisola Salentina, e i monti dell' $\Lambda$ ppenuino Jucano. Ijsturbato essenzialmente da scaglie tettonicle e sovascorrimenti limitati, ed occasionalmente da faglie isolate con direzione appenuinica er antiajpenninica.

Distretto 9 - ReTrordese APpExxixico - Comprendente gli edifiei vuleanici dell'Amiata, Vulsini, Cimini, Sabatini, Vuleano Luziale e Flegreo.

Je faglie si presentano con andamenti appenninici ed antiappenninici. In questo distretto va incluso anche l'ajplauto vuleauico di Roccamonfina.

Distretto 10 - Araxp.tese appexsixico - Comprendente la fossa Bradanica e quella Abruzzese-Narchigima, fino allavanpaese Romagnolo.

Le formazioni sono costituite dalla serie calcareo-gessoso-solfifera e dai ricoprimenti marginali della Marnoso-i renacea.

Distretto 11 - MIssiccio Garcixico - Costituito prevalentemente dalle formazioni calcaree, in facies Abruzzese.

Distretto 12 - MURGF - Predominanza telle formazioni calcaree, superficialmente nessuna purticolare deformazione tettonica.

Distretto 13 - Massicci CaLabro-Pelonisaxo - Puo essere indicato come un massiccio cristallino ovunque sovasscorso su baciui ti sedimentazione periferiea.

Distretto 14 - Sicilia Settentisoxale mo Ormentale - Comprendente le formazioni alcareo-dolomitiche con i sovrascorrimenti dei Monti di Palermo e le zone di affioramento del flyseli paleogenico. Iu questo distretto è compresa anclie la piattaforma Iblea.

Distretto $7 \dot{j}$ - SICluia Occioexrale - Caratterizzata dal taxolato di Ragusa, e dalle formazioni argillose-arenace-manose e dalla caleareo-gessoso-solfifera.

Distretto 16 - Busso Thnexo - Unia regione italinna curaterizzata dai terremoti profondi, essa è sede anche di vulcanismo attivo ed è seguata da discontinuità che penetrano fiuo alla "astenosfera".

Distretto 17 - SARDEGa - Costituente il massiceio Sarto-Corso, fa parte di un antico bloceo cristallino notevolmente stabile. 


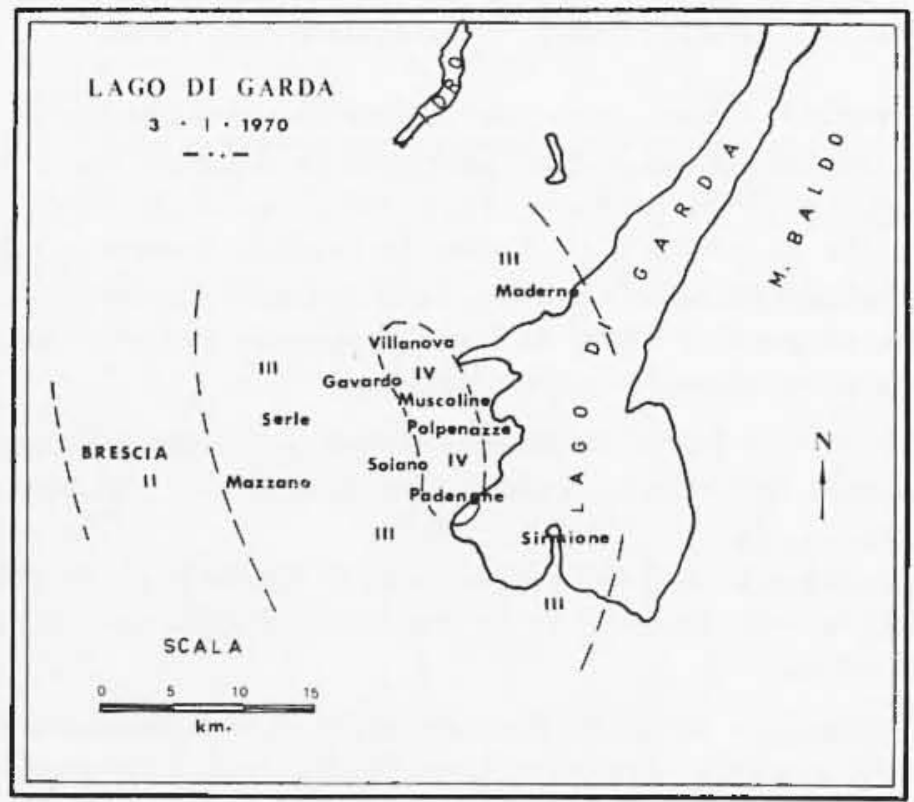

Fig. 3 - Le linee tratteggiate indicano l'andamento delle isosiste. Le località interessate dal $\mathrm{IV}$, si trovano su depositi morenici e fluvio-glaciali, o lungo i bordi delle formazioni calcares liassiche.

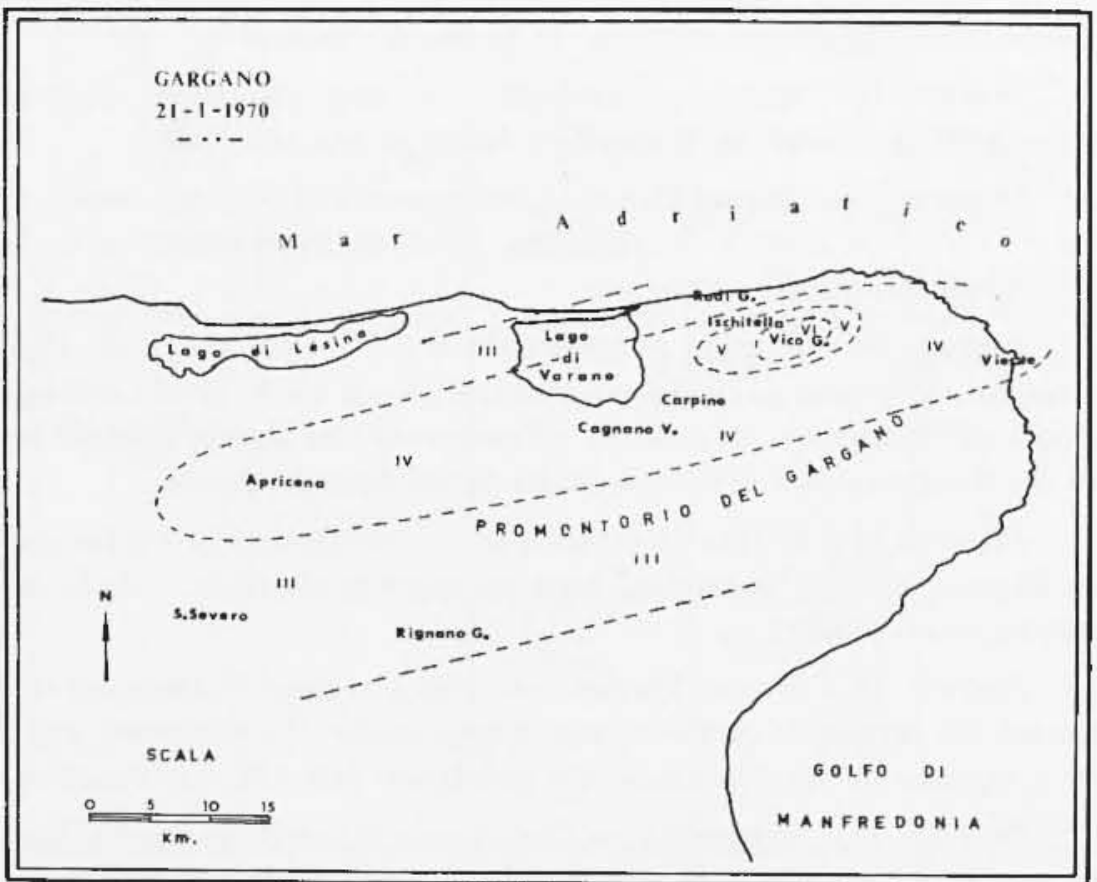

Fig. 4 - Landamento dello isosiste ì nguale allat direzione delle faglic

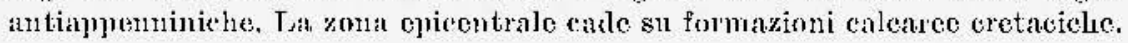




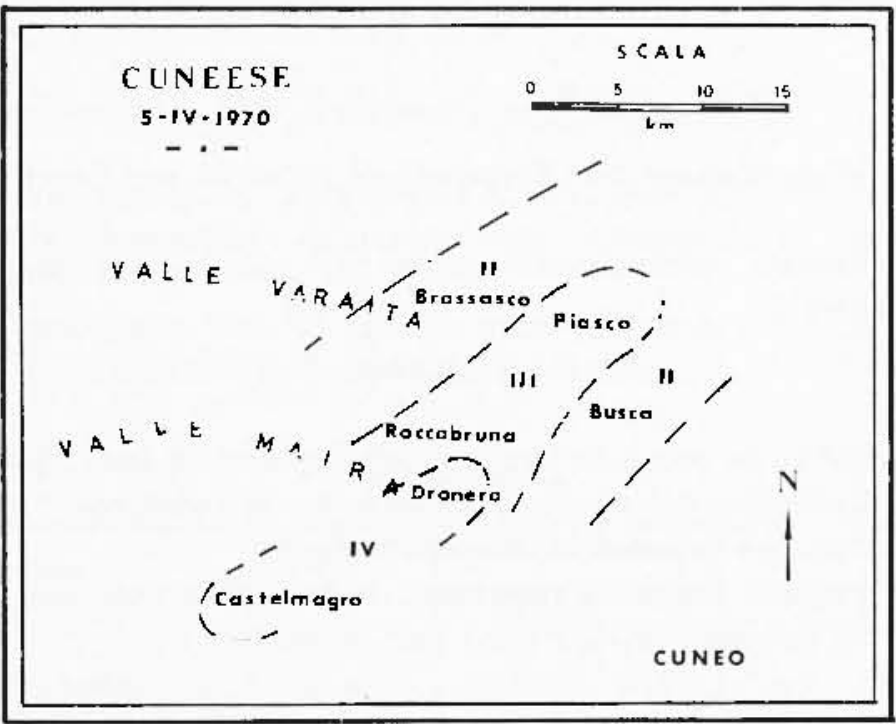

Fig. $\check{a}$ - Lespicentro è localizzate snlle formazioni calcarec tel Trias sup. Le isusiste sono inclicate dalle linee trattegriate.

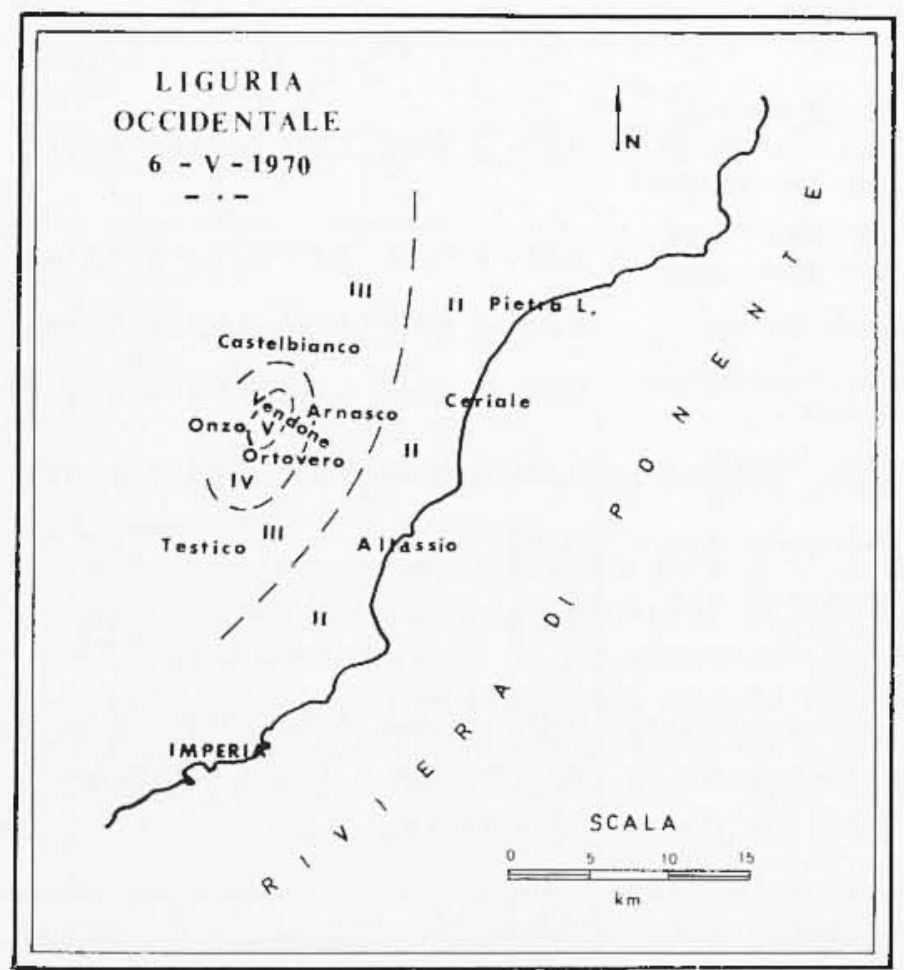

Fig. (j - La zona interessata dal sisma è il fronte delle Penniti, Je localiti più colpites sono sulle formazioni calearec ti Albenga. 
Formule di comelazione tra Magnitudo ed Intensiti per $i$ vari distrotti

La formula comunemente usata per calcolare la magnitulo macrosismica i:

$$
M^{*}=a I_{o} \div b \log _{\mathrm{g} 0} h+c
$$

in cui $h$ intiea la profonditì ipocentrale espressa in $\mathrm{km}, \mathrm{i}$ parametri $a, b, e$, dipentono tal tlistretto sismico clie si consitlera, l'intensiti all'ipocentro $I_{o}$ ì espressa in Merealli-Sieberg.

Per maggior comoditi riportiamo la tab. 1 , in cui sono indicati i valori dei parametri $a, b$, o per i vari distretti.

TABELA I

\begin{tabular}{|c|c|c|c|}
\hline J)istretti & $a$ & $b$ & $c$ \\
\hline$\left\{\begin{array}{l}1-\Lambda l_{p i} \text { Marittime } \\
2-A l_{p} \text { Oecilentali } \\
3-\Lambda l_{j} \text { Seltentrionali }\end{array}\right.$ & $0,674 \div 0,054$ & $1,35^{2} \pm 0,099$ & $1,091 \pm 0,213$ \\
\hline$\left\{\begin{array}{l}4-\Lambda p_{p i} \text { Merislionali } \\
5-\Lambda l_{p} \text { Meridionali }\end{array}\right.$ & $0,437+0,050$ & $0,874 \pm 0,190$ & $0,714 \pm 0,373$ \\
\hline 6 - Valle Padana & $0,484 \pm 0,079$ & $1,040 \pm 0,210$ & $0,324 \stackrel{-1}{-1} 0,272$ \\
\hline $\begin{array}{c}7 \text { - App. Ligure-Tosco- } \\
\text { Romag. }\end{array}$ & $0,437 \pm 0,050$ & $0,874 \pm 0,190$ & $0,714 \pm 0,373$ \\
\hline $\begin{array}{l}8 \text { - App. Imbro- } \\
\text { IIarch.-Abruz. }\end{array}$ & $0,421 \pm 0,048$ & $1,138 \pm 0,146$ & $0,179 \pm 0,178$ \\
\hline 9 - Retropacse $\Lambda$ pp. & 0,467 & 一 & 0,800 \\
\hline$\left\{\begin{array}{l}10-\text { Avanpaese App. } \\
11 \text { - Cargano } \\
12 \text { - Murge }\end{array}\right.$ & 0,472 & - & 1,2 \\
\hline 13 - Calabro-]'eloritano & $0,414 \doteq 0,063$ & $1,105 \pm 0,201$ & $0,367 \pm 0,251$ \\
\hline 14 - Sicilia Orientale $\left({ }^{*}\right)$ & $M \quad 0,024 I^{2}{ }_{n-1}$ & $0,206 I_{0}+2,15$ & 7 (per $I_{0} \geqslant \mathrm{VII}$ \\
\hline 15 - Sicilia Occ. $(*)$ & $M=0,481 I_{o}$ & 1,407 & $\left(I_{0}<\mathrm{V} I \mathrm{I}\right)$ \\
\hline
\end{tabular}

$\left(^{*}\right)$ L'assenza di dati sufficienti ci consiglia per ora l'impiego delle formule ricavate dalla Marcelli (14). 
Sismicità delle Alpi Harittime e zone adiacenti.

Da una nota di M. Bossolasco e C. Eva pubblicata sui rendiconti della XII Assemblea Generale della Commissione Sismologiea Europea (?).

Riportiamo in Tal, 2, l'elenco delle principali scosse e dei dati and esse relativi quali risultano dalla Tab. 1 (?).

TABELA 2

\begin{tabular}{|c|c|c|c|c|c|c|c|c|}
\hline & \multirow{2}{*}{ Data } & \multicolumn{3}{|c|}{ T.M.G. } & \multicolumn{2}{|c|}{ Coorilinate } & \multirow{2}{*}{$\begin{array}{c}\text { Prof. } \\
h\end{array}$} & \multirow{2}{*}{$M_{1}$} \\
\hline & & h. & $m$ & $\varepsilon$ & $\varphi$ & $\lambda$. & & \\
\hline & Febbraio & 12 & 07 & $4(6,5)$ & $+4^{*} 29^{\prime}$ & $\mathrm{S}^{\circ}+2^{\prime}$ & 9,8 & 3,00 \\
\hline & Mar\%o & 17 & 45 & 09,9 & $44^{\circ} 18^{\prime}$ & $7^{\circ} 31^{\prime}$ & 6,2 & 2,40 \\
\hline & Marzo & 02 & 17 & 07,8 & $44^{\circ} 30^{\prime}$ & 7,14 & 7,2 & 2,57 \\
\hline & Aprile & 07 & 29 & 35,0 & $44^{\circ} 04^{\prime}$ & $7^{\circ} 40^{\prime}$ & 3,9 & $3,2 \overline{1}$ \\
\hline & Aprile & 18 & 53 & $3 I, 8$ & $44^{\circ} 19^{\prime}$ & $7^{\circ} 20^{\circ}$ & 11,3 & 3,21 \\
\hline & Maggio & 09 & 16 & 57,6 & $44^{\circ} 07^{\prime}$ & $8 \circ 06^{\prime}$ & 11,2 & 3,41 \\
\hline & Giugrio & 23 & 21 & $06 ;, 8$ & $44^{\circ} 32^{\prime}$ & $8^{\circ} 39^{\prime}$ & 7,6 & 3,33 \\
\hline & Luglio & 13 & 11 & 27,1 & $44^{\prime \prime} 14^{\prime}$ & $7^{\circ} 23^{\prime}$ & 4,9 & 2,21 \\
\hline & Ottobse & 22 & $5 \mathrm{I}$ & 16,0 & $43^{\circ} 34^{\prime}$ & $7^{\circ} 35^{\prime}$ & 3,5 & 3,50 \\
\hline & ottome & 22 & 33 & I 8,0 & $43^{\circ} 53^{\prime}$ & $8025^{\prime}$ & 8,1 & 2,53 \\
\hline
\end{tabular}

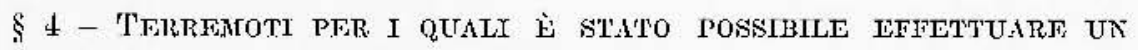
ESAME DETTAGILATO

Sono stati trattati dettagliatamente quei terremoti risultati piu significativi e attendibili.

La deserizione verte su tre punti; la prima parte si riferisce ai dati analitici ricavati ed alle determinazioni efletturte da altre Organizzarioni. Successivamente è elencata la fenomenologia matcrosismuca con la descrizione delle isosiste el è riportato anche il coefliciente di estinzione dell'intensita marosismiea in superfieie. Segue ancora nuta breve descrizione geologico-tettonica delle zone colpite dall'evento sismico.

ATTENUAZIONF PELA'LNTEXSTTA MACROSISMTCA IN SUPERTICTF Per questo grmp) di terremoti dei quali avevamo notizie più esatte 
sono state disegnate le isosiste e successivamente abbiamo calcolato il coefficiente di estinzione dell'intensità macrosismica in superficic.

Il coefficiente is stato ottenuto tramite la relazione

$$
a=-\frac{2,30}{D-h} \log _{-\infty} \frac{I_{0}}{I}
$$

in cui, a rapjresenta il coefficiente di estinzione, $D$ la distanza ipocentrale $\left(D=\sqrt{r^{2}+h-}\right), h$ la profondità ipocentrale, $I_{o}$ è l'intensità all'epieentro, $I$ quella alla distanza $r$ dall'epicentro.

Il coefficiente $\alpha$ è stato calcolato per le direzioni ritenute più significative dal punto di vista geologico.

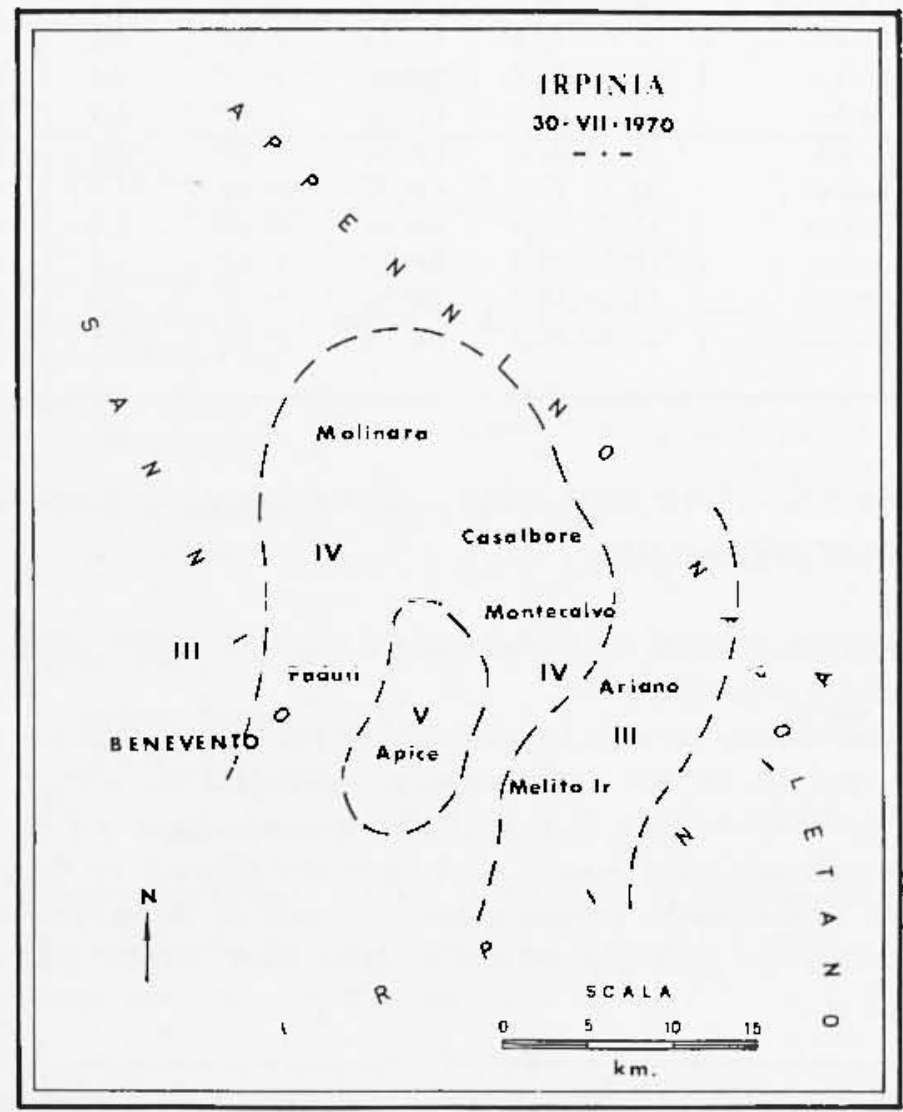

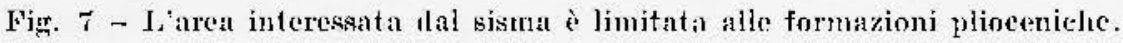




\section{PRINCIPATI THREAIOTI AVVENUTI SETA'ANNO}

1) - 9 Febhaio - Aprexino Forzivese

$H_{0}=07$ '39"08s; $\varphi=43{ }^{\circ} 58^{\prime} ; \lambda=12^{\circ} 06{ }^{\prime} ; h=28 \mathrm{~km} ; M=4,5$ (RALP)

$I_{0}=\mathrm{V}^{\top} \mathrm{I}$

Altre determinazioni epicentrali

(U.S.C.G.S.) $H_{0}=07^{13} 39^{\mathrm{m}} 12 \mathrm{~s}, 7 ; q=44,4^{\circ} ; \lambda=11,7^{\circ} ; M=4,4 ; h=\mathrm{N}$ (B.C.I.S.) $\quad H_{0}=07^{n} 39^{\mathrm{n} 10} \mathrm{~s} ; \quad \phi=44,0^{\mathrm{n}} ; \lambda=11,8^{\circ}$

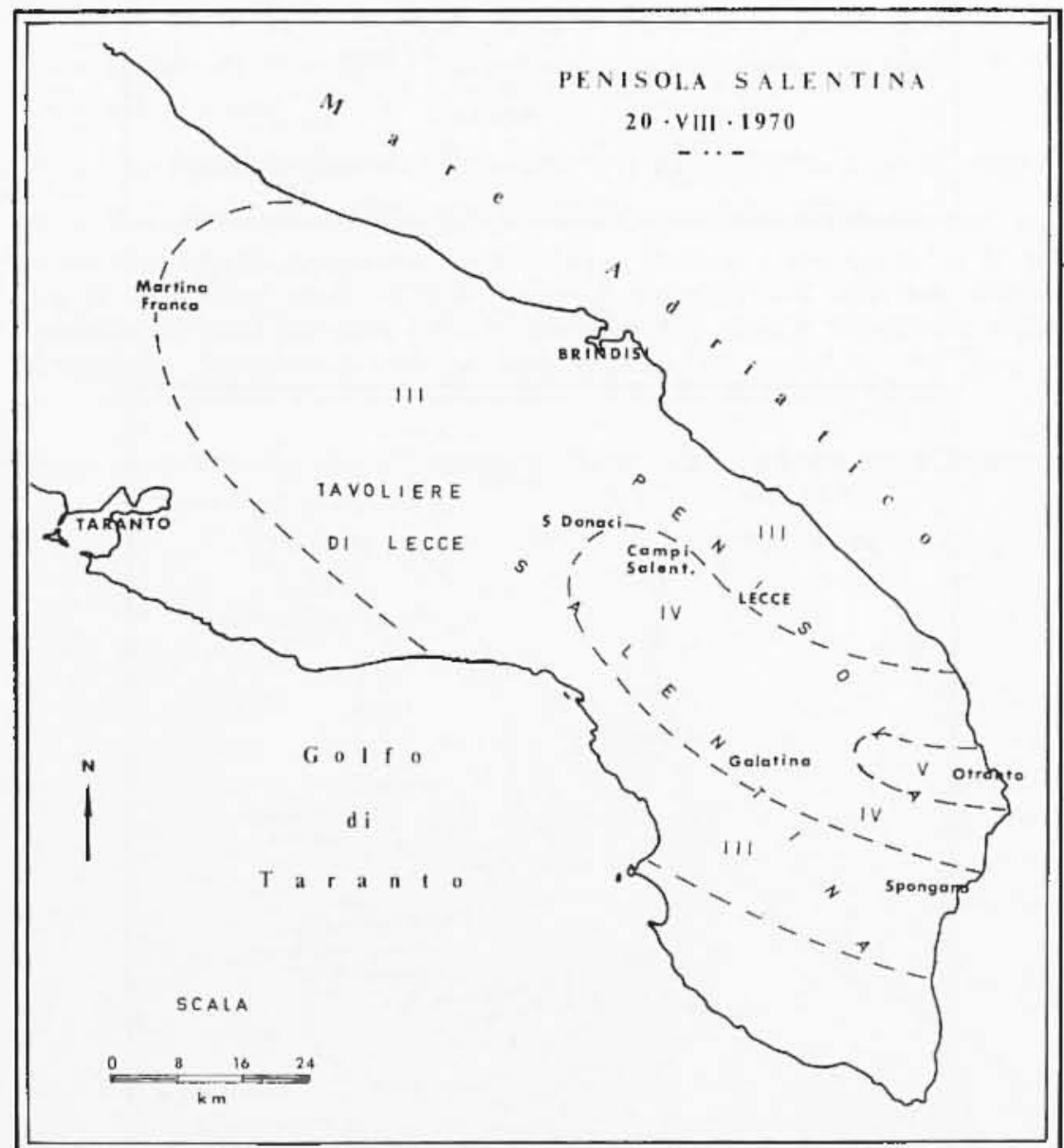

Fig. s - Le isosiste sono allungate nella direzione elelle frimeipali facrlie (direzione appenuiniea). Le zome magriormente interessate thall'erento sono sulle formazioni caleareo-arenatec minceniche. 


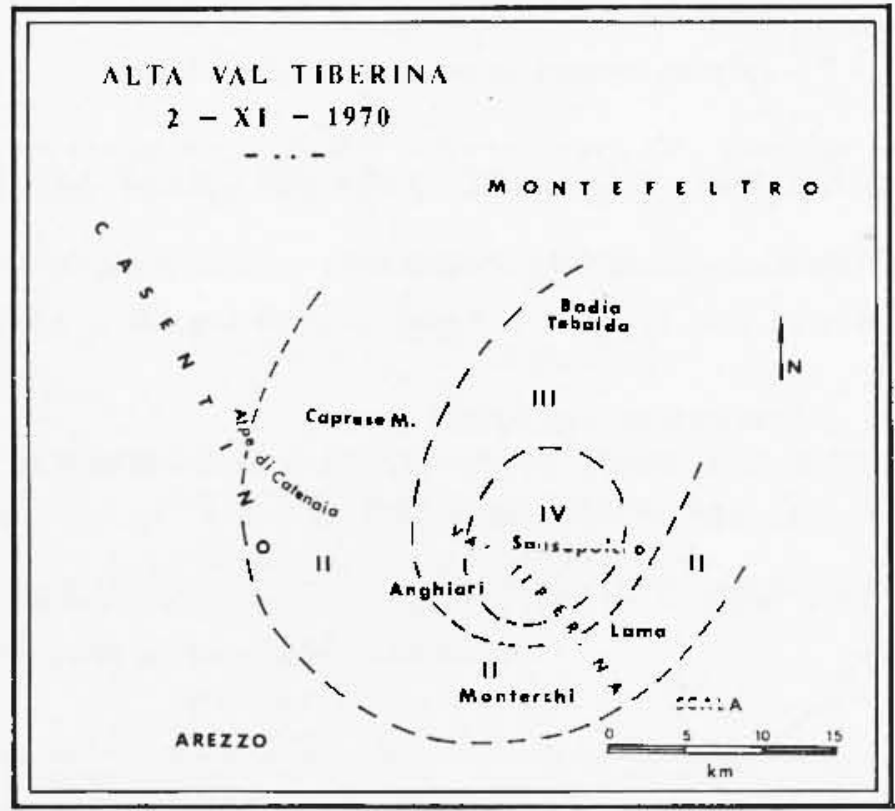

Fig. 9 - Le isosiste hanno un andamento tragversale all'asge del bacino lacusire dell'Alta Val Tiberina. L'epicentro si loealizza nella zona di passaggio tra flyscle eocenico, e formazioni del bacino larustre (sulssirtente).

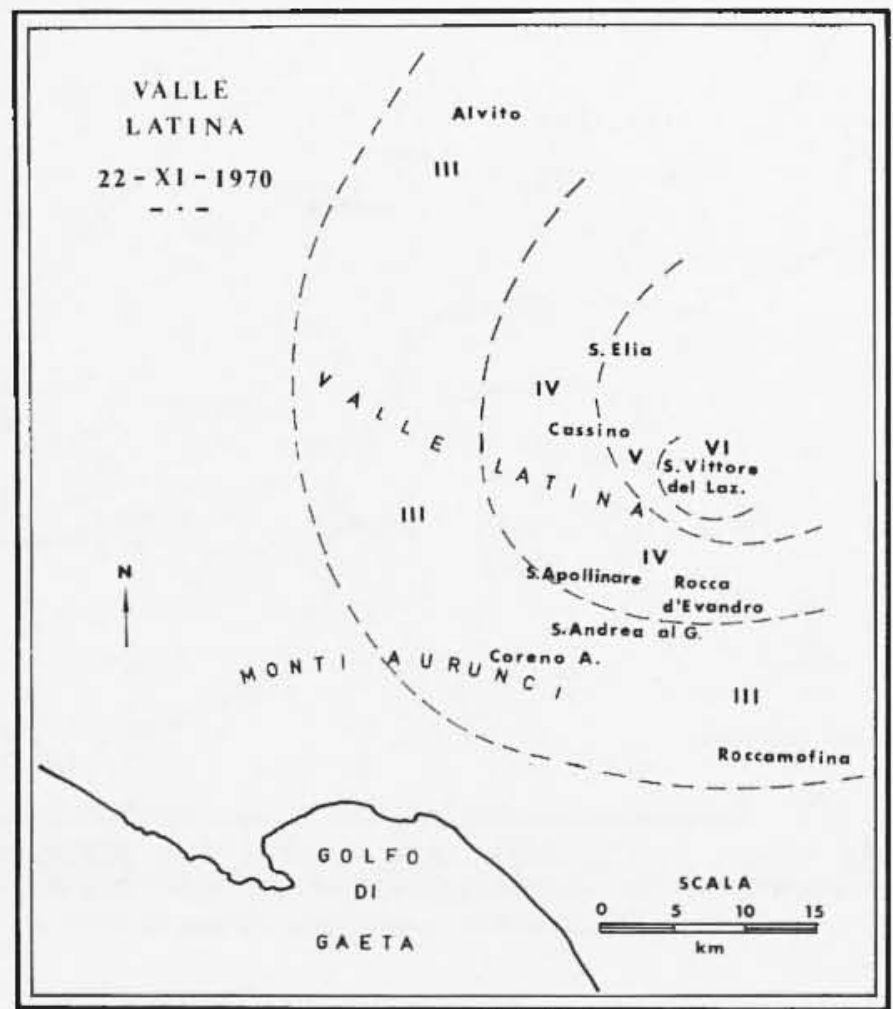

Fir. 10 - Lo isosiste presentano una forte attenuazione in eorrispondenza dei massieci calcarei Mesozoici e dell'apprarato vulcanico di Roccamonfina. 
Notizie macrosismiche.

Questo terremoto si is sviluppato turante un breve periodo sismico. Si sono registrate, quattro seosse premonitrici, tra le $05^{\mathrm{h}} 1 \mathrm{i}^{\mathrm{m}}$ e le $06^{\mathrm{h}} 41^{\mathrm{m}}$ circa, con intensiti tra il II edl il III grado. Lá scossa principale è stata seguita da due repliche, alle ore $08^{n}{ }^{\circ} 1^{\mathrm{m}}$ compresa tra ii IV-Y grado, ed alle $09^{n} 52^{\mathrm{m}}$ sentita di III.

L'intensità macrosismica in superfieie si è manifestata nel seguente modo:

VT a Civitella di Romagna, Galesta, Roeck S. Caseiano, S. Sofia;

V a Bagno di Romagna, Morligliana, Premilenore, Tredozio, Borgo S. Lorenzo;

IV a Dovadola, Meldola, Barberino nel Mngello, Scarperia, Prato;

III a Castrocaro-Terme, Terra del sole, Fiorenzuola, Cesena, Sarsina, Montespertoli.

Le isosiste (Fig. 11) del VI gralo, sono tutte contenute nella formazione Marnoso-drenacea Romagnola. Le localiti che lanno

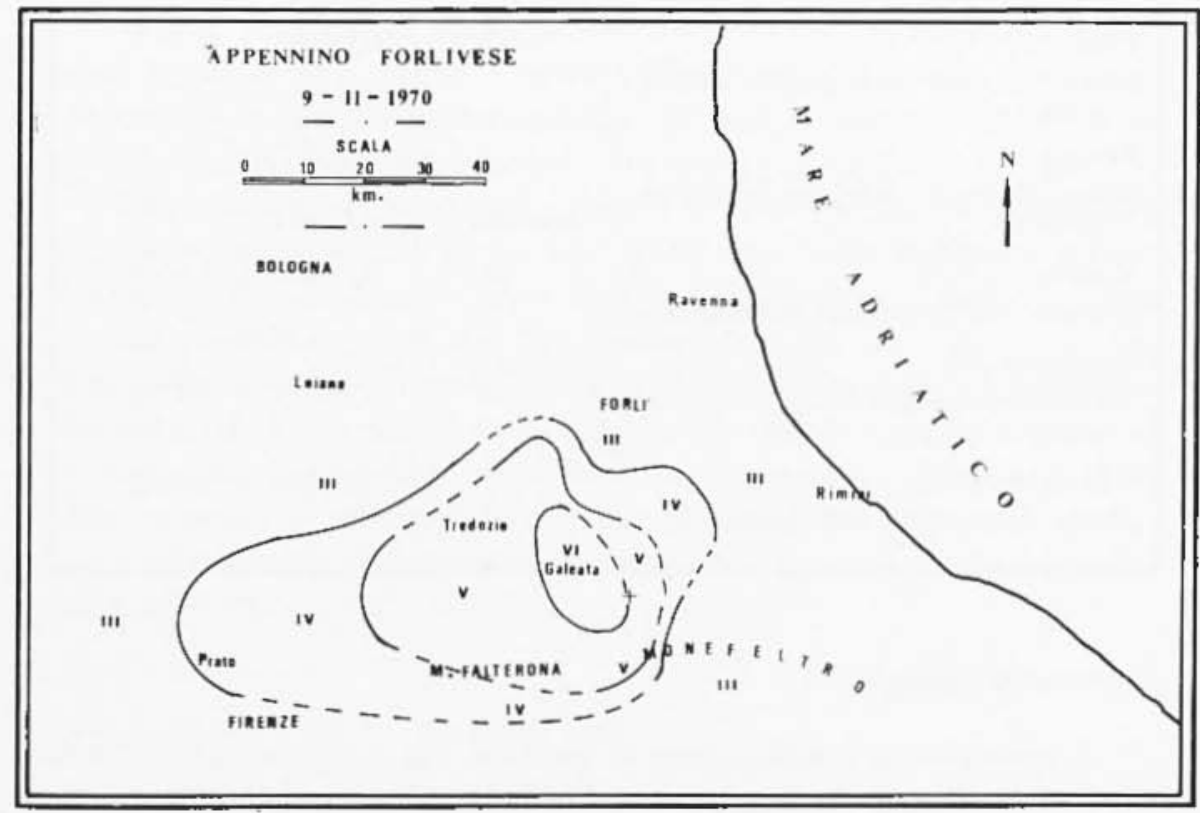

Fig. 11 
sentito il sisma di VI grado, sono tutte contenute tra la piega-faglia di Palazzuolo al il limite tettonico tra il flysch Iarmoso-Arenaceo (Elveziano) ed il flysch Arenace-3larnoso (Tortoniano), o si allineano luugo un asse di sinclinale. Tale isosista si presenta in forma ellitica, allungata verso NW-SE.

Le altue isosisto di $V$ e IV grado, tendono a limitarsi lungo la fascia bordiera Panlana, ad occidente si prolungano fìno ad oltrepassine la dorsale del M. Falterona, iuteressando il Bacino Fluvio-Tacustre del Mugello. Si manifestano in maniera anomala con un $V$ a Borgo S. Lorenzo e duo IV a Senperia, Barberino. A spiegazione di questo fatto, si uota che nel Bacino del Jugello i depositi Villafranchiani, non sono orizzontali o suborizzontali, ma inclinati verso $\mathrm{N}-\mathrm{TV}$, a causa del probabile sollevamento relativo alla sponda di N-E.

Nella tab. 3 sono riportati i valori del coefficiente di attemazione.

TABELIA A 3

\begin{tabular}{|c|c|c|}
\hline Ijirezione dall'epiecntro & $\begin{array}{l}\text { ])istanzal } \\
\text { lin11 }\end{array}$ & $\begin{array}{l}\text { Valnre del enellisionte } \\
\text { di attenuazionc }\end{array}$ \\
\hline $\begin{array}{l}\text { Prato } \\
\text { (Parallelamente alle pieglic dell'ap- } \\
\text { peninino) }\end{array}$ & $3 \overline{5}$ & 0,01082 \\
\hline $\begin{array}{l}\text { Prato } \\
\text { (Parallelanente allo piogho doll'ap. } \\
\text { pennino) }\end{array}$ & successivi & 0,00871 \\
\hline $\begin{array}{l}\text { Rumini } \\
\text { (Fronte ilollavanfossa Mareligiana) }\end{array}$ & 26 & 0,04400 ․․ 0,0043 \\
\hline 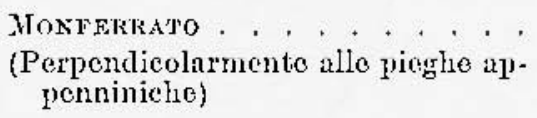 & 20 & 0,05301 上 0,0102 \\
\hline 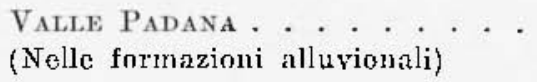 & 31 & 0,02361 : 0,0058 \\
\hline
\end{tabular}

\section{Lineamenti geologici}

L'epicuntro di questo sisma si localizza sul versante palauo dello Alpe di s. Benedetto (appenuino Forlivese). Precisamente in quella fasciat compresa tra le pieghe apjumniniche e lo pieghe sub-padane. 
Con essa coincidono anche $\mathrm{i}$ più attivi centri sismici dell'intero $A]$ jenennino settentrionale.

In grenerale lo stile tettonico della regione i costituito da pieghe e pieghe-faglie arenti tiratone SWF-SE, e vergenti a oriente. Questo motivo è interessato da due sistemi di faglie compressive, parallele e subparallele allo pieghe stesse. E da notare che nella zona epicentrale mancano le faglie tlistensive, posteriori alla fase plicativa tellorogenesi(*).

\section{2) - 28 Feburaio - Pozztom}

A partive tal 28 febbraio di quest'anno la zona di Pozzuoli (Napoli) è stata interessalat tat un'intensa at tivita microsismica. Durante l'anno, dalle stazioni appositamente installate a cura dell'Osservatorio Vesuviano e del C.N.R. (laboratorio per la Geofisica della Jitosfora) sono state registante 1600 scosse ciron.

Tale attivita ì tovuta alla accentuazione del fenomeno bratisismico negativo. Storiemente si riscontra dal II see. A.C. al $\mathbf{X}$ see. un bralisimo positivo, tall'XI sec. al XVII secolo braclisimo negativo, tal XVII sec. ai primi del XX bralisimo positivo o attulanente nua nuova inversione de] fonomeno.

Questi movimenti epirogenetici sono dovuti a fenomeni più 0 meno legati al vulanismo Flegreo ed Ischitano. Tue soosse dhe hamno eartterizzato questa mierosismieiti, in genere erano di piccola intensità, con gri ipocentri compresi tra 0 e $1 \mathrm{~km}$, e tra 1 e $5 \mathrm{~km}$.

Seconto Penta $\left(^{15}\right)$ ed altiri Autori, il tetto rel bacino magmatico giace ad una profonditio di $\$-5 \mathrm{~km}$. Nella zona della Solfatam invece, il complesso vuloninico-serlimentario ragriungereble i $2000 \mathrm{~m}$ ciren.

La probabile earasa del bratisismo (17) i da attribuirsi alle variazioni del livello del marma nol focolaio, le fat ture vuleano-tet toniche ble suddividono in zolle il tetto del focolato stesso, quando avyengono le variazioni precodenti, provocano il sollevarsi e l'abbassarsi thelle zolle causando il aratteristico fanomeno bralisismico.

Riassumiamo jer completezza quanto è riportato nella relazione del C.N.I. ( $\left.{ }^{6}\right)$.

(*) Bollettino I.N.G. - Febbraio 1970.

Bollettino U.S.C.C.S. e B.C.I.S.

Scrvizio Geologieo - Carta Geologiea d Italia 1:100.000, Foglio 99 "Faenza" 1969 - e relative note illustrative. 
Gli epicentri tlell'attuale periodo sismico, si allineano seconto tre tirettrici, sempre parallele alle zone di frattura gia esistenti, o alle faglie con impostazione profonda. Due allineamenti sub-paralleli tra loro ed alle faglie con impostazioni profonda, hamo direzione NNVY-SSE. Il primo prossimo alla costa oceidentale del golfo di Pozzuoli e passante per Baia, Valtro spostato più ad Est, e passante per 3. Nuovo. Lin terzo allineamento ancom più ad list dei precedenti, e con direzione WNW-JSE passante per st. Nowvo e J'ozznoli. it la notare che un adtensamento di epicentri si ha nellatrea di Pozzuoli e nella zona tlella Solfatarit.

Il fenomeno della Solfatara può essere considerato come locale rispetto al fenomeno generale del processo bradisismico (*).

3) - 19 Aprile - LAGO DI GARDA

$H_{o}=18^{\mathrm{n}} 16^{\mathrm{m}} 32^{\circ} \mathrm{s} ; \varphi=45^{\circ} 39^{\prime} ; \dot{\lambda}=10^{\circ} 28^{\prime} ; \quad h=13 \mathrm{~km} ; M=4,3$ (RRP) $I_{o}=\mathrm{VI}$

Altre determinazioni epicentrali

(B.C.I.S.) $H_{v}=18^{\mathrm{n}} 1 \mathrm{j}^{\mathrm{m}} 37^{\mathrm{s}} ; \varphi=45,8^{\mathrm{n}} ; \lambda=10,4^{\circ} ; \not h=0$;

B.N.S. - VHF. $M=4,1-$ MON. $M=4,2$

\section{Notizie macrosismiche}

L'attuale sisma ha interessato le Prealpi Itombardo-Venete, si è manifestato prevalentemente lungo la riva oceidentale del Lago di Gorda.

Il terremoto è stato avvertito di:

VI a Gavardo, Voloarno, Villanova sul Clisi;

V a Serle, Garda, Gardone Riviera, Salò, Toscolano;

IV a Caprino Veronese, S. Ambrogio in Val Policella, Bardolino, Brescia, Desenzano, Sirmione, Iimoue sul Garda, Padenghe sul Garta, Malcesine, Mazzano, Costermano, Tivoli, Macesine;

III a Sesto S. Giovanni, Peschiera del Garda, Milano, Lonato, Serravalle all'Adige, Borgosatollo, Cavrina, Carjenedolo, Verona, Castelnuovo di Verona;

(*) Bollettino I.N.G. - Febbraio 1970.

Servizio Geologico, Carta Geologica d'Italia $1:$ 100.000, Foglio 183-184 "Isola d'Ischia - Nipoli n, 1967. 
II a Paxia, Torrevecellia Pia, Montieclli Pavese, Chignolo Po, Corsieo, Como, Casalolilo, Roverbella, Gazoldo regli Trpoliti, Serravalle a Po.

Le isosiste sono riportate in Fig. 12.

Le localita interessate dal VT grado si allineano lungo la direzione NNE-SSO, direzione delle faglie giudicariesi. Queste località poggino su sedimenti fluvio-glaciali e alluvionali (ghiaie ex argille). Tali formazioni se di grande potenza smorzano l'onergia sismion per attrito interno, mentre nelle zone marginali tenolono ad esaltare le sollecitazioni.

Le isosiste verso $\mathrm{N}$ \& $\mathrm{NW}$, ciò verso le Austridi, s'infittiscono, questa zona costituita prevalentemente cla Greis e Micascisti intensamente fagliati la un comportamento a bloceli rlisgiunti e rallentano fortemente la propugazione ilellenergia macrosismica. Un aspetto simile anche se in seala minore, lo presentano le Alpi Calcaree Heridionali, direzione di NW.

La direzione di massima propatgazione si riscontra lungo la Valle Padana, con andamento E-WV ('lab. 1).

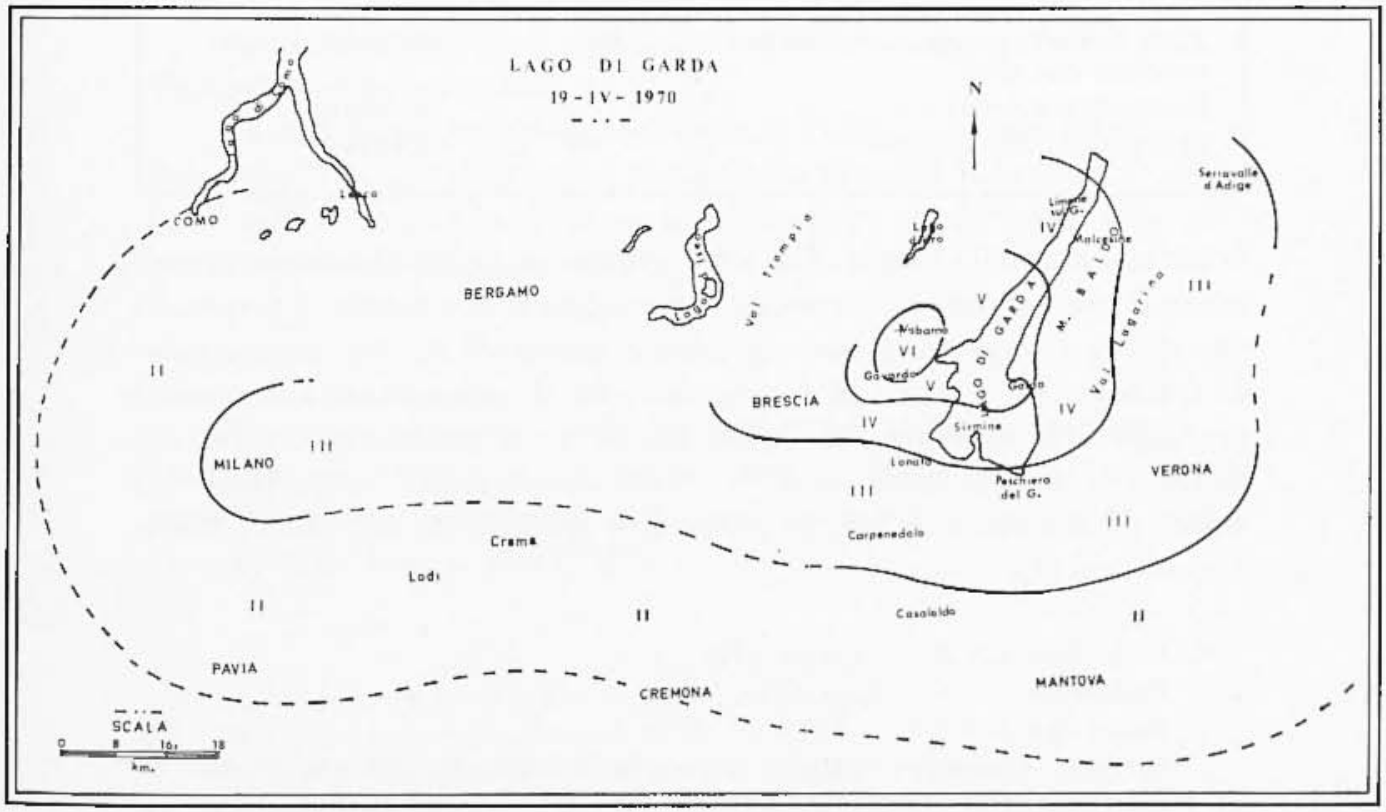

Fig. 12 


\section{Tettonica generale}

Jat zona tetitonich, responsabile dell'attuale sisma is guella compresa tra la parte meridionale delle Gindioure e l'anticlinale di XI. Jaldo. Isb zona in generale è costituita da una snecossione di pieghe e sovrascorrimenti con direzione NE-SW.

Je faglie, in generale derivano dalla rottura dei fianchi intermedi delle pieghe, (pieghe-faglie), sono di tipo inverso, e presentano lo stesso

$$
\text { TABELA } 4
$$

\begin{tabular}{|c|c|c|}
\hline Direzione tall'epicentio & $\begin{array}{l}\text { Distanza } \\
\text { km }\end{array}$ & $\begin{array}{l}\text { Valore del coefliciente } \\
\text { di attenuazione }\end{array}$ \\
\hline $\begin{array}{l}\text { Verona e Iropagini moridionali } \\
\text { delle Alpi ealeuree }\end{array}$ & (60) & $0,01546 \pm 0,009$ \\
\hline $\begin{array}{l}\text { Parallelamente alla fascia lor- } \\
\text { diera petana entro il limite delle } \\
\text { risorgive verso oecidente }\end{array}$ & 8 & 0,08040 \\
\hline $\begin{array}{l}\text { l'arallelamente alla faseia bor- } \\
\text { diarl pardana entro il limite delle } \\
\text { risorgive verso oceidente }\end{array}$ & 26 & 0,01880 \\
\hline $\begin{array}{l}\text { Parallelamente alla faseia hor- } \\
\text { diera padana entro il limite delle } \\
\text { risorgive verso occidente }\end{array}$ & 60 & 0,00708 \\
\hline $\begin{array}{l}\text { Sud: fino alle propargini delle for- } \\
\text { nnazioni calearec }\end{array}$ & & $0,0.5352$ \\
\hline De positi noreniei & & $0,0.4040$ \\
\hline Formazioni l'lioteniothe & 40 & 0,012383 \\
\hline
\end{tabular}

orientamento delle pieghe. Cn altro gruppo di faglie di minore importanza si presentino con direzione subortogonale allo pieghe. I terremoti che qui si manifestano sono in genere provocati ala nu meceanismo di frattura. Jes profonditi ipocentrali che si riscontrano, sono molto prossime alla snperficie di discontinuiti della crosta, tra strato tlel Ganito a strato intermenlio (10). Tale discontimuita nella zon del Gartab $\left(^{5}\right)$, ì a circa $15 \mathrm{~km}$, la profontita ipocentrale del sismub attuale ì di $13 \mathrm{~km}(*)$.

(*) Jollettino I.N.G. - Aprile 1970.

Bollettino U.S.C.G.S. - 1970.

Bollettino B.C.I.S. - 1970.

Servizio Geologico - Carta Geologiéa d'Jtalia I : 100.000, Foglio 34 "Breno " 1970 - foglio 47 "Brescia " 1968 - foylio 48 "Pesclicera del Gartil 1969 - e relative note illustrative. 
4) - 29 Giugno - Piaxa de Catanzaro

$H_{u}=14^{\mathrm{n} 22 \mathrm{n} 52 \mathrm{~s} ;} ; q=38,9^{\circ} ; \lambda=16,6^{\circ}\left({ }^{*}\right) ; h=\geqslant 10 ; M=3,2$ (MMP)

$I_{0}=\mathrm{IV}$

Altre determinazioni epicentuali

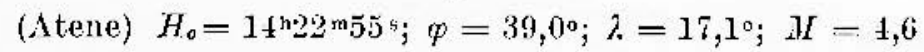

(I.N.G.) $H_{0}=1+2.2 \mathrm{~m} 52 \mathrm{~s}$

Notizie macrosismiche

Questo sisma si è manifestato nella piana di Catanzaro, è stato

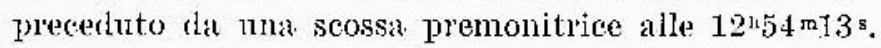

E stato sentito di:

IV a Vazzano, Pentone, Sorbo S. Basile;

III a Beleastro, Soveria Mamnelli, Platonia, Magisano, Taverna, Ottanzaro, Andali;

II a S. Floro, Lamezia Terme, IIarcellinara, Petrona, Feroleto Antico.

Rispetto a come si è manifestata l'energia macrosismica, il $\mathrm{IV}^{\top}$ di Vazzano risulta anomaio.

La debole propagazione dell'energia sismica ha reso incerte l'isosiste (Fig. 13). Il III gralo si è manifestato nelle zone cleila Piana di Catanzaro o lungo i bordi di essa. II II grato si riscontra nelle parti meridiomali rlelle incisioni vallive.

\section{Tettonica}

La zona epicentrale cade su formazioni quatemario-plioceniche, costituite da congiomerati, marne, argille. Questa zona, esclusa la parte orientale è contornata dalle roce formanti il basamento cristallino (graniti, gneiss, filladi) (**).

(*) Jce coordinate sono state calcolate grafieamente.

(**) Bollettino I.N.G. - Gingno 1970.

Bullettin Sational Observatory of Athene - 1950 .

Servizion Geologieo - Carta geologiea d'Italia . 1 1.000 .000 - Roma 19651 . 


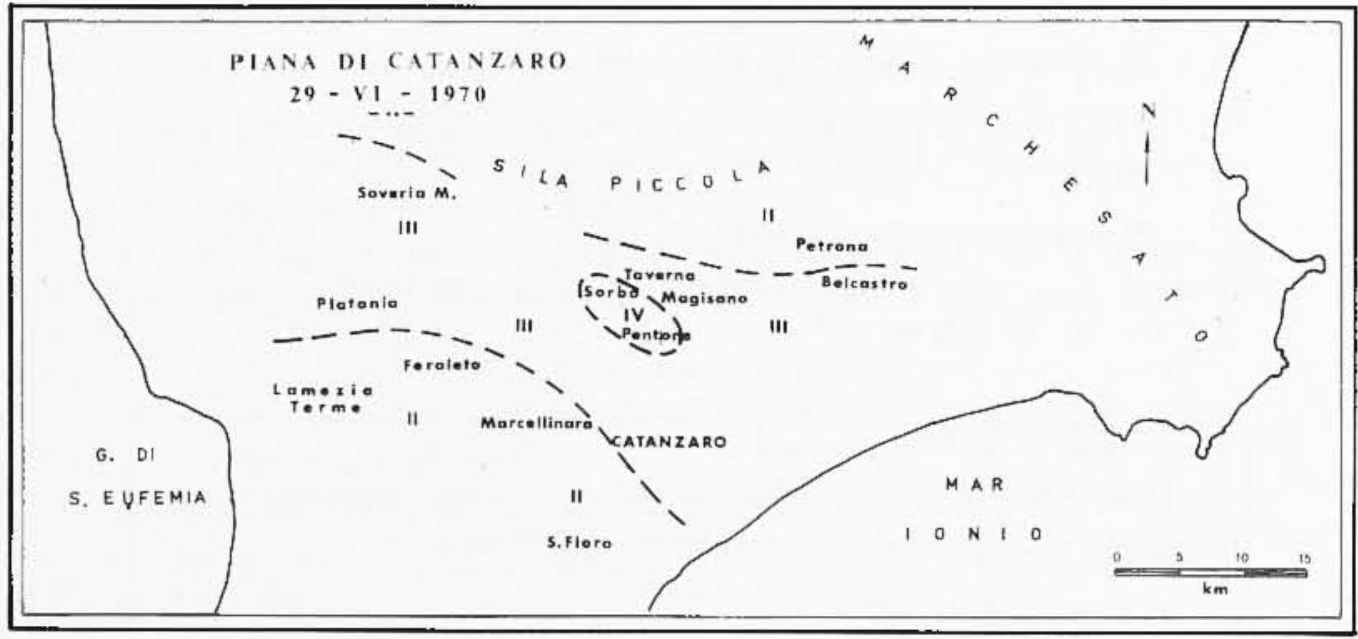

Fig. 13

5) - 7 Luglio - VaI Nenisa

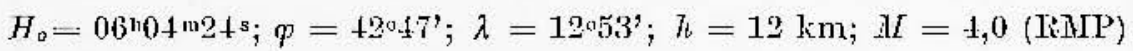

$I_{0}=\mathrm{V}$

Altre determinazioni epicentrali

(C.S.C.G.S.) $I_{a}=06^{14} 04^{m} 21^{\mathrm{s}} ; p=42,8^{\circ} ; \lambda=13,0^{\circ} ; h_{b}=17 \mathrm{~km}$;

$M= \pm, 0$

(B.C.I.S.) $\quad \Pi_{0}=06^{10} 04^{\mathrm{m}} 24^{\mathrm{s}} ; \phi=42,8^{n} ; \lambda=12,8^{n}$.

\section{Notizie macrosismiche}

Il sisma ha interessato lu Val Serina, e le zone al essa circostanti manifestanılosi tli:

$\mathrm{V}$ a S. Anatolia di Narco;

IV a Campello sul Clitumno, Caseia, Spoleto;

III a Monteleone di Spoleto, Sellano, Trevi;

II \& Monte Custello Vibio, Torli, Montecastrilli.

Una replica è seguita alle $0 y^{\mathrm{n}} 32 \mathrm{~m} 23 \mathrm{~s}$. 
Te isosiste presentano un andamento coneentrieo alquanto regolare, a dimostrazione di una uniforme propagazione dell'energia macrosismica in superficie, fig. 14 .

Il coefficiente d'estinzione dell'energia macrosisnica in superficie is compreso tra 0,03439 e 0,04505 . Questi valori, sono validi in ogni direzione e per distatuze comprese tra $16 \mathrm{~b}-25 \mathrm{~km}$ dall'epicentro. Sella direzione appenninica si hat $\bar{a}=0,03264 \pm 0,0043$ per pereorsi fino a $1.6 \mathrm{~km}$.

Le formazioni alluvionali e lacustai, come quelle della Valle UTbrat, della Valle del Tevere, e della Piana di Terni, non hanno dato forme di esaltazione dell'energiat in superficie. Itat profondits ipocentrale di $12 \mathrm{~km}$ circa, colloca il sisma nello strato del Granito, che qui raggiungerebbe i $2 \breve{5}-30 \mathrm{~km}$ di potenza ( $\left(^{5}\right)$.

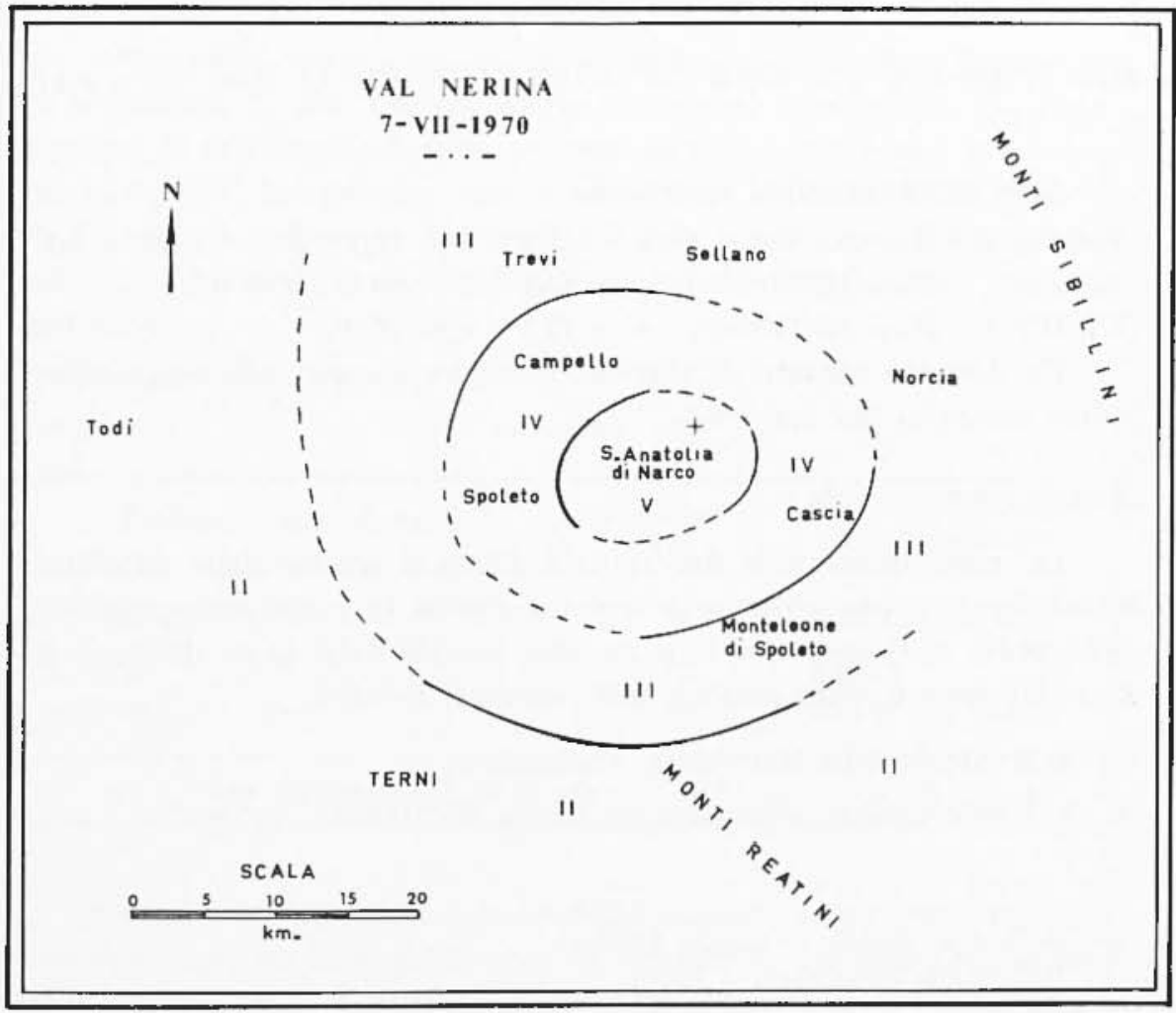

Fig. 14 


\section{Considerazioni Geologiche}

La zona interessata dal sisma è la fascia di pieghe tlellar regione Umbra, parallele e vergenti verso l'Alriatico. Esse hamo direzione NNE-SSW, e costituiscono parte tella virgazione Sabina. La regione ¿̀ caratterizzata da nua tetioniea a pieghe con continui fenomeni disgiuntivi. Abbiamo ampie anticlinali e strette sinclinali. L'ejuicentro si localiza sul versante orientale dell antielinale di Honte S. SilvatoreMonte Galenne. Quest'anticlinale ha tirezione N-S fino alla zona di Sellano poi piega verso $S W$, per breve tratto, e riprente infine la dlirezione iniziale. I'anticlinale si presenta con il suo fianco orientale rovesciato ed interessato da numorose faglie con andamento parallelo all'asse dell'anticlinale, in alemi la struttum viene interrotta da faglie trasversali inverse (*).

6) - 19 Agosto - Colline rueidllifere

$$
\begin{aligned}
& H_{o}=12^{\mathrm{v}} 19^{\mathrm{n}} 46^{\circ} ; \varphi=43^{\mathrm{n}} 05^{\prime} ; \lambda=10^{\mathrm{n}} 46^{\prime} ; \quad h=10-1 . \mathrm{km} ; \quad M=4,1 ; \\
& I_{0}=\text { VI }
\end{aligned}
$$

Altre tleterminazioni epicentrali

(U.S.C.G.S.) $H_{0}=12^{11} 19 m 54, \mathrm{~s} 5 ; \varphi=43,2^{\circ} ; \lambda=11,1^{\circ} ; h=\mathrm{N} ; M=5,1$

(B.C.I.S.) $\quad H_{0}=12^{\mathrm{n} 19^{\mathrm{m}} \tilde{0}^{2} \mathrm{~s} ;} \varphi=43,0^{\mathrm{n}} ; \lambda=10,8^{\circ} ; \hbar=0$

(I.N.G.) $\quad H_{0}=12^{\mathrm{h}} 10^{\mathrm{m}} \mathrm{50} \mathrm{s} ; \quad \varphi=43,1^{\circ} ; \lambda=10,9^{\mathrm{n}}$.

Un disereto numero di stazioni sismiche assegna alla magnitudo valori compresi tra 4,4 e 4,7 .

\section{Notizie macrosismiche}

La zona interessata dall'attuule sisma è quella delle "Colline Hetallifere w, e particolaumonte colpita risulta la parte settentrionale della prov. thi Grosseto, e la parte merilionale tlelle prov. di Siena e Pisa. Il sisma è stato sentito nelle seguenti località:

VI a Monterotondo Marittimo, Pomarance;

V a Monteseudaio, Guardistallo, Massa Mauttima;

(*) Bollettino [.N.G. - Juglio 1970 .

Bollettino U.S.C.C.S. - 1970 .

Bollettino B.C.I.S. - 1970.

Servizin Geologieo - Carta Geologien d'Italia 1 : J00.000 - foglin 13 I

"Foligno" 1968 - foglio 123 " Assisi " 1969 - e relative note illusirative. 
IV a Volterra, Saline di Voltera, Montieri, Roccastrada, Suvereto, Ponte Ginori, Cecina, Riparbella;

III a Castellina Marittima, Capannoli, Grosscto, J'iombino, Follonica, S. Vincenzo, Donoratico, Kanglinno di Grossedo, Rosignano M., Castagneto $\mathrm{C}$;

II a I'isa, Livorno, I'alaia, MEontipoli, Firenze.

Le isosiste (Fig. 15) si presentano con forma ellittical allungate lungo la direzione $\mathrm{N} \backslash \mathrm{V}-\mathrm{SE}$, testimoniano una quasi uniforme attenuazione dellenergia macrosismica in superficie, lungo la fiscia di IV grato, le isosiste si limitano ad interessare le sole Colline Metallifere.

I valori rlell'energia macrosismica sono riportati in tab. õ.

\section{Tettonica della zona}

L'ejicentro viene a catere, nel complesso delle Colline Metallifere, in prossimiti di una sorgente di gas, di natua idrotermale. La distribuzione di tali manifestazioni arviene di preferenza lungo le fratture intersecanti il basamento, esse in superficie ajpaiono come serie di rughe e dossi, ed hamno direzioni NW-SE, e sono spezati in piccole zolle da fratiure trasversali principalmente, e longitudinali secondariamente.

TA IILLA 5

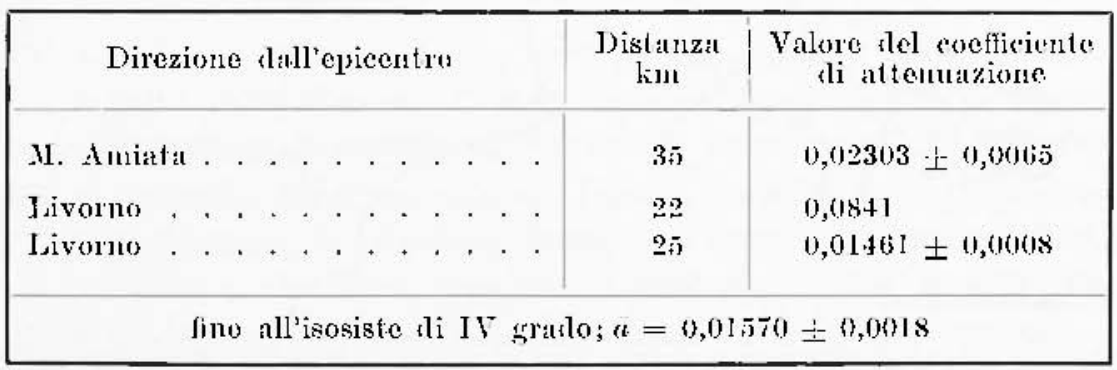

Ja serie stratigrafiea tella regione ì molto complessa, infatil si hamo rinque suecessioni diverse di tereni, di mi quattro formate in bacini setimentari tlisfinti. Tella zona epicentrale la serie toscana si presenta continu fino alla formazione tlel "macigno". Ter tale serie, in cuesta zona si ha nn accumulo nom indifferente della formazione 


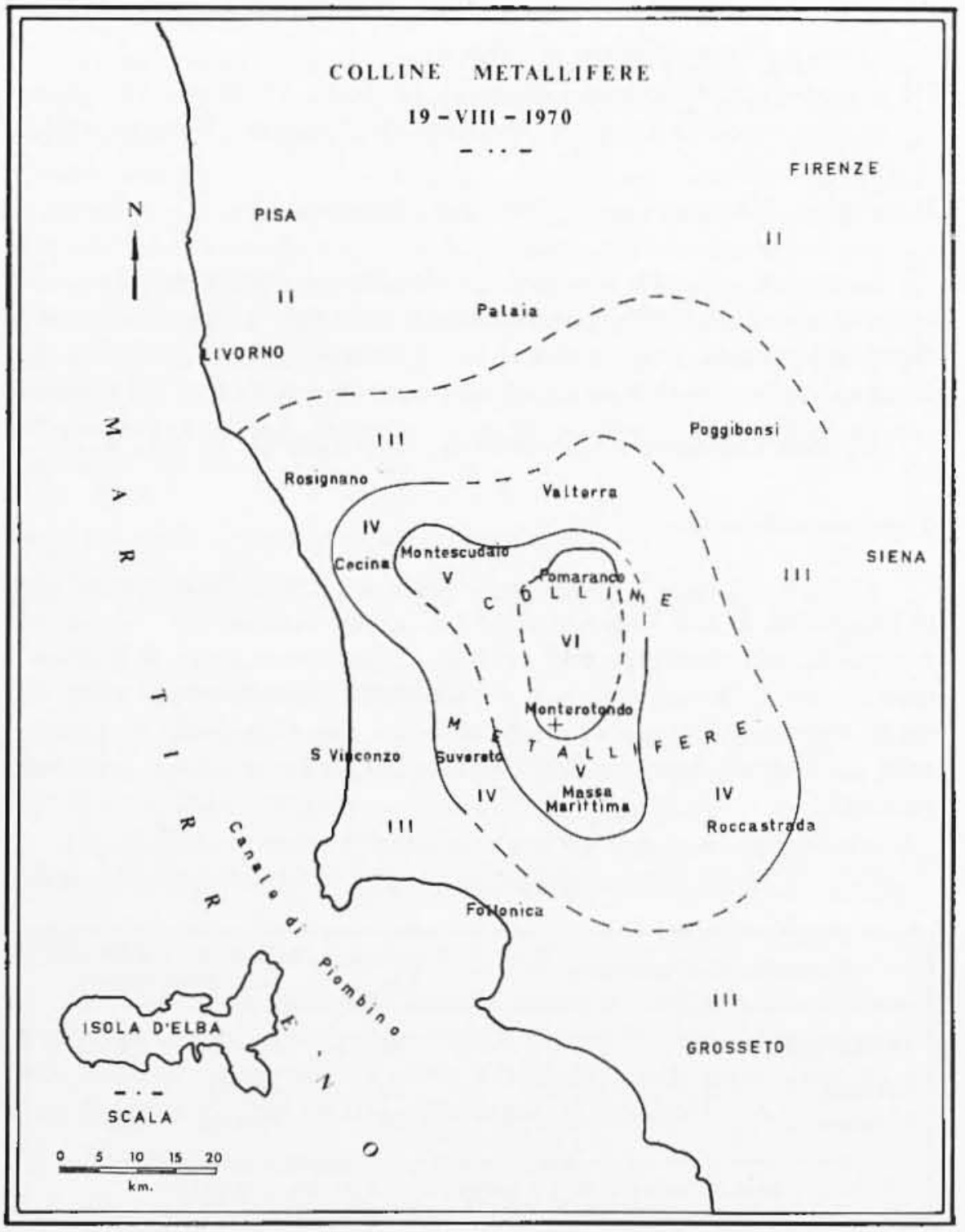

Fig. 15

evaporitica del Trias sup. Ricordiamo che tale serie ha un comportamento plastico, tlovuto agli strati tli anilrite, ed è la serle dello scollamento tra basamento e copertum. 
Conseguentemente allo scollamento si è avula la taralazione di alcune aree con conseguente accumulo della formazione evaporiticat (Monterotondo Marritimo).

Risulta altresi che il basamento corrisponde ad un basso strulturale, mentre lat copertua forma un alto strulturale.

L'ipotesi sull'origine delle manifestazioni di vapore in questa zoma (regione Toseana), è da attribuirsi all'esistenza nel sot.tosuolo di "plutoni granitici di rifusione $"\left({ }^{18}\right)$.

L'origrine del sisma è legata alla natura di tali manifestazioni idrotermili (*)).

7) - 7 Settembre - ApPexisixo Unвко

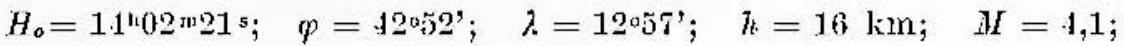

$I_{0}=\mathrm{VI}$

Altre deteminazioni epicentrali

(U.S.C.G.S.) $\quad H_{0}=1 \frac{1}{2}\left(02 m 23, \mathrm{s2} ; \varphi=43,0^{\circ} ; \lambda=12,9^{\circ} ; \quad h=36 \mathrm{~km}\right.$; $M=4,3$

(B.C.I.S.) $\quad H_{0}=14^{\mathrm{h} 02 \mathrm{~m}} 23^{\mathrm{s}} ; \quad \varphi=13,0^{\mathrm{a}} ; \lambda=12,6^{\circ}$.

Notizie macrosismiche

Il sisma si è manfestato nella regione Umbua interessando le segruenti zone:

YI a Sellano;

$Y$ a Fiastra, Pioraco;

IV a Falriano, Genga, Apiro, Belforte sul Chienti, Matelian, Campello sul Clitumno, Cascia, Foligno, Spoleto, Trevi, Arrone, Ferentillo, M[ontefranco, Stroneone, Terni, Sefro, s. Severino;

III a Maceata, M[agliano Sabino, Aneona, Ascoli P'iceno, Colmomano, IIonteleone di Spoleto, Perugra, Pesaro, Contigliano, Rieti, Teramo, Camerino, Gatgliola, Castel Raimondo, Fiordimonte;

II a Castellino, Jesi, Brontesanvito, Penna S. Giovanni, Montefalco, Postr.

(*) Bollettiono I.N.G. - Agosin 1970 .

Bollettino L.S.C.G.S. - 1950 .

Bollettino B.C.I.S. - 1970.

Servizio Geologieo - Carta Geologiea d'ltalia $1: 100.000$ - foglio 119

"Massa Marittima 1909 - e relative note jllustrative. 


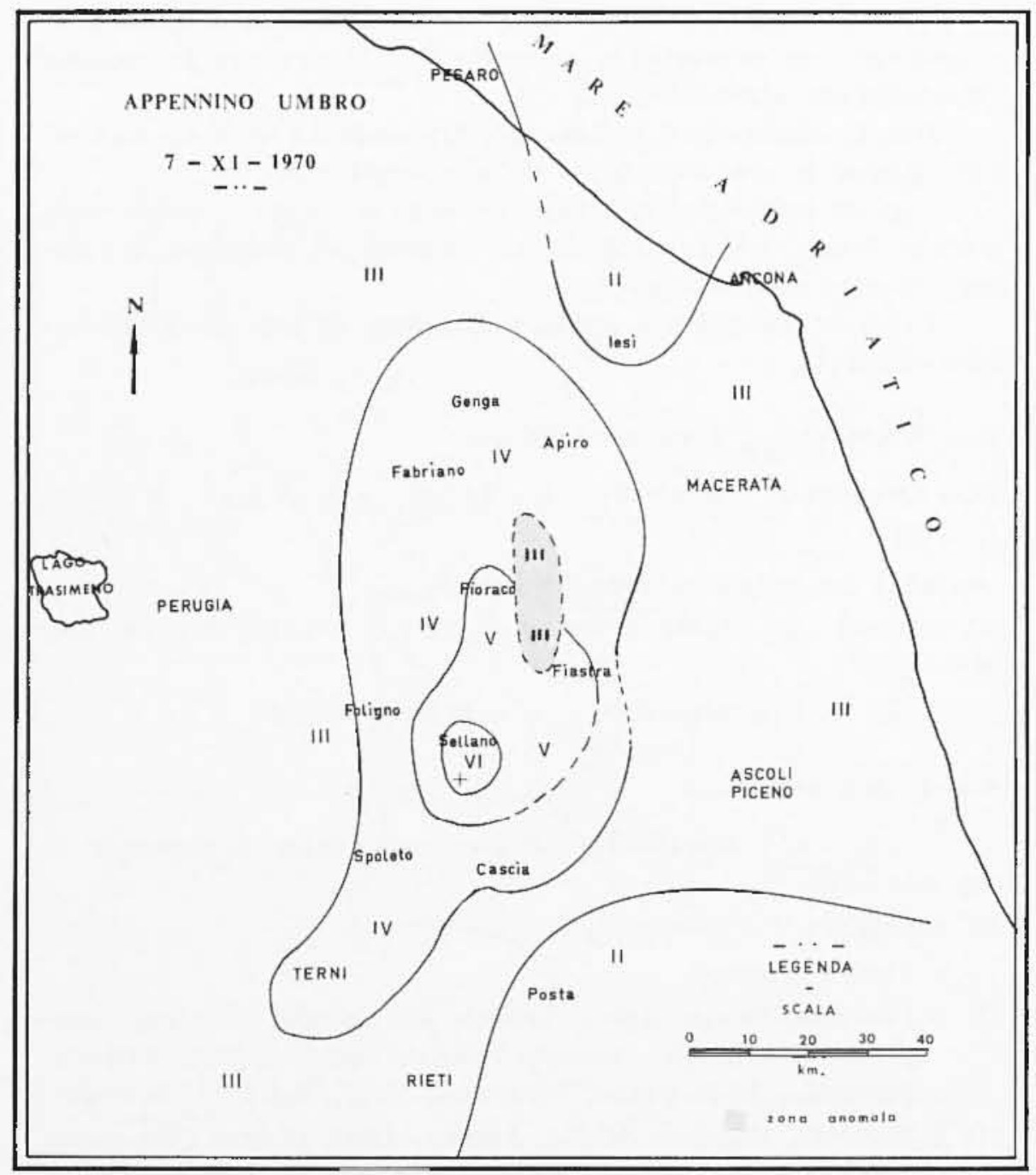

Fig. 16

E secruita una repplica alle $16^{\mathrm{h}} 1 \mathrm{t}^{\mathrm{m}} \mathrm{m}(\mathrm{s}$ s.

Je isosiste (Figr. 16) si presentano allungate con direzione N-S, NE-SW. Presentano maggione antpiczan verso l'Adriatico e sono contenute nella formazione Cmbro-Marchigrana fino al IV grado. Il III grado ì in genere oltre det ta formazione ed interessa terrani recenti. Di rilievo e'è da notare una zona anomala tra il V eal il IV grado, in 
cui il sisma is stato sentito rli III. Tale zona si identitica con la sinclinale di Camerino, il eni asse ha direzione $\mathrm{N}$ W-SSE. Il riempimento di

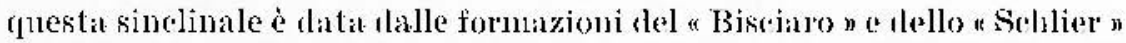
e da molasse. Nlla forte potenza di questa formazione $(1000 \mathrm{~m}$ ciuca), va astribuita l'attenuagione dell'energia sismica. The anomalie gravimetriche di Bouguer, in questa zona lanno valori intorno allo zero,

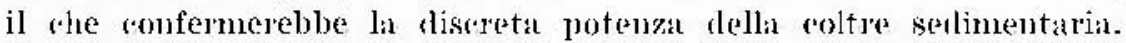
Nella tab. o sono ripostati i valori del coefficiente ali attenuazionc.

T A R E L L L A (F

\begin{tabular}{|c|c|c|}
\hline Direzione tallepicent ro & $\begin{array}{c}\text { Distanza } \\
\text { kull }\end{array}$ & $\begin{array}{l}\text { Valore del coofficionte: } \\
\text { di atemuazional }\end{array}$ \\
\hline . . . & $31 ;$ & 0,00778 \\
\hline Noril . . . . . . . . . . & 45 & 0,00017 \\
\hline $\begin{array}{l}\text { CAscia } \\
\text { (Perpendicolarmente alle pieghe ap- } \\
\text { pentuniche) }\end{array}$ & 9 & $(1,0772)$ \\
\hline $\begin{array}{l}\text { Cascia } \\
\text { (Perpendicolatmente alle pioghe alp- } \\
\text { penninioho) }\end{array}$ & 21 & $0,035601 \pm 0,000053$ \\
\hline Orest. . . . . . . . & 20 & $0,05+114 \pm 0,0088$ \\
\hline
\end{tabular}

\section{Lineamenti tettonici}

L'attuale morfologin si is delineata con le lasi recenti della tettonica post-miocenica. it dat precisare clue nel pliocene la formazione di pieghe d dovuta principalmente alla muggiore subsidenza delle sinrlinali rispetto alle anticlinali. Per eui i movimenti verticali diliorenziati prevalgono sui movimenti tangenziali. Te notizie geologiche losnite per il terremoto del $f$ Iuglio sono valide anche per l'attuale, lata la vicinanza degli epicentri e la stessa natura geologico-tettonica (*).

(*) Bollettino I.X.G. - Settembre: 1970 .

Bollettino [C.s.c.(t.s. - 1970).

Bollettino B.C.L.S. - 1970.

Servizio Cieologrico - Carta Goologica d Italia I : 100,000 - foglio 131 a folignon 1968 ... fogglio 124 "Macerata * 1969 - c relative note illustrative. 
8) - 27 Settembre - MIgNixo MonTe LuNGo

$H_{0}=19^{\mathrm{n}} 18^{\mathrm{m} 30 \mathrm{~s}} ; \not=41^{\circ} 22^{\prime} ; \lambda=14^{\circ} 01^{\prime} ; \hbar=7 \mathrm{~km} ; M=3,9(\mathrm{RMP}) ;$

$I_{0}=\mathrm{VII}$

Altere determinazioni epicantrali

(U.S.C.G.S.) $H_{0}=19^{\mathrm{n} 18} \mathrm{n} 36,7^{s} ; \varphi=41,7^{\circ} ; \lambda=13,8^{\circ} ; \hbar=27 \mathrm{~lm}$

(B.C.I.S.) $\quad H_{0}=19^{\mathrm{n} 1} 18^{\mathrm{m}} 32 \mathrm{~s} ; \quad \varphi=41,2^{\circ} ; \lambda=14$, (1॰.

\section{Notizie merosismiche}

La zona di Mignano Nonte Lumgo (prov. di (asserta), è stata interessata da un hugo periorlo sismico con eirea 800 scosse nell'treo di sei mesi. La prima di queste scosse ha ragginnto il VIl grado, delle altre solo 16 lamino superato il IV grindo. Y'elenco tlelle seosse di maggiore intensitic is stato riportato in Tab. $\tau$.

T'A IS J. I. A T

\begin{tabular}{|c|c|c|c|c|c|c|c|}
\hline Data & Incalità & $\begin{array}{l}\text { T.Ml.ct. } \\
h \text { in. s }\end{array}$ & $\begin{array}{c}\text { Coorit. } \\
p\end{array}$ & $\begin{array}{c}\text { Eipicent. } \\
\lambda\end{array}$ & $h$ & If & $I_{a}$ \\
\hline G. $\mathrm{X}$ & Mignano M.L. & 06551 & $+1^{\circ} 22^{\prime}$ & $1+t^{\circ} 01$ & 7 & 3,0 & $v$ \\
\hline $31-x$ & Rescamonfina & 011624 & $41^{\circ} 22^{\circ}$ & $14^{\circ} 01^{1}$ & 5.6 & 3,4 & V.VI \\
\hline $21 \cdot X$ & & $0736+10$ & $+1^{\circ} 22^{\prime}$ & 14001 & 6 & 3,4 & V.VI \\
\hline $22 \times 1$ & s. Vittore & $23+715$ & $+1 " 22^{\prime}$ & $14^{\circ} 01^{\prime}$ & 6; & 3,5 & VI \\
\hline $26-\mathrm{XI}$ & ล. Pietro Infine & 035719 & $+1^{\circ} 22^{\circ}$ & $1+\div 01:$ & 6 & 3,2 & $\mathrm{v}$ \\
\hline $25-\mathrm{XII}$ & Migrnano Y.L. & 0214 & $41^{\circ} 22^{\prime}$ & $14^{\circ} 01^{\circ}$ & $5 \cdot 6$ & 3,2 & $\mathrm{~V}$ \\
\hline
\end{tabular}

11 sisma ò stato sentito ali:

VII a Mignano Monte Lmugo, Cave, Campozillone, Conea della Campania, Piantoli, Starza;

VI a Galluccio, Inrzano Appio, Tora, Piecilli;

$r$ a San Pietro Infine, Grottola, Caspoli;

IV a Vairano Patenora, Vairano Saalo, Caianello;

III a Roco al'Fvandro, 3I. Cesima, Presenzano, Pietravairano, Tuoro, Fontanafredia, Cocuruzzo. 


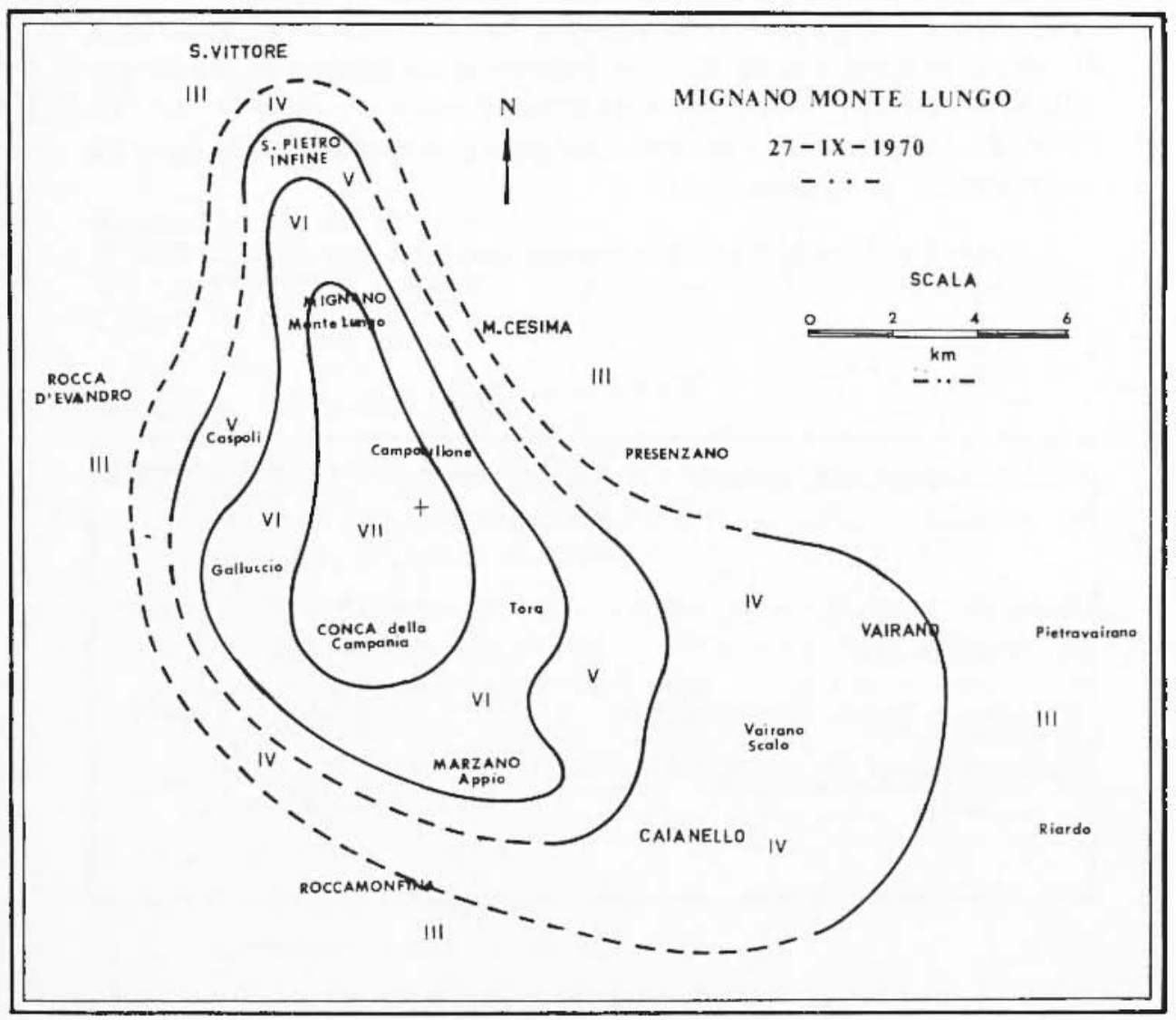

Fis. 17

Io isosiste sono riportate in Fig. 17.

L'amlamento delle isosiste, mette in evidenza la piccola profonditit. ipocentrale che ha aratterizzato il sisma e che i compresa nel primo strato della rrosta. Si suppone che per questa zoma, M. Cesima, una prima superficie di discontinuiti si abbia a circa $10 \mathrm{kms}\left({ }^{8}\right)$. Data la gravimetria della zom non si eschudo che essa possa essere la comad.

Le isosiste si presentano nolto allungate lungo lat valle del Percia, in direzione dela quale si ha la massima projagazione lellenergia in superficie. I'apparato vulesuico di Toceamonfina, ed i massicei 
malaurei li Houte Cesima e di Roeca l'Evandro, si sono comportati come tegli ostacoli alla propagazione di tale energia in superficic. In alcune zone in a si averano formazioni eterogenee a diversi costituenti litologici (lave, tufi, argille), si riscontra un'esaltazione rell'energia. In generale si ha peró una rapida attenuabiono dellenergia macrosismicu in supericeic.

I valori del roefficiente di attenuzione sono riportati in Tab. 8.

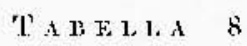

\begin{tabular}{|c|c|c|}
\hline 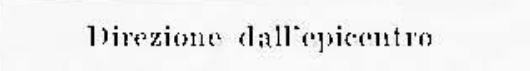 & $\begin{array}{l}\text { Joistauzal } \\
\text { limi }\end{array}$ & $\begin{array}{l}\text { Valore del coelliciente } \\
\text { di attenuabione }\end{array}$ \\
\hline Valle del Jencia . . . . . . . & 8 & וה \\
\hline Valle del P'sucia & 15 & 0,05795 \\
\hline 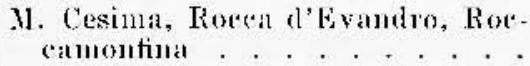 & 6.8 & $0,01+153 \pm 0,063667$ \\
\hline 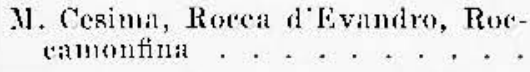 & 2 & $(1,12525+(1,01855)$ \\
\hline
\end{tabular}

Considerazioni yeologichs

Lo cauatleristiche sismiche della regione consirlerata, cioè degli Aumei a Fort ed i Monti S. Croce, Magrgiore, Rocernonfiua a Sul, comprentono due tipi di eventi sismici: quelli dosuli alla tedtonica risgiuntiva e compressiva di natua post-orogenetica, del retropaese anjenninico, e quelli dovuti all'apparato clello "strato vulcano" ali Roccamonfina.

La tetonica ó quella del retropaese appenninico e dellappennino autoctono. Si lanno grossi allineamenti di faglia dirette sui versanti orientali erl inverse sui versanti occiclentalli. Olfre a questi tipi di faglie che hanno direzione appenminies, ve ne sono talte di miose importanza rispetio alle precedenti, le quali lanno direzione antiappenninica (YE-SW). 
All'intersezione di questi ordini di faglie, nella Valle del Peccia in frossimita tell'abitato di Mignano Moute Lumgo, si ì avuta la localizzazione tegli ipocentri tellat tale perioto sismieo (*).

\section{9) - 29 Settembie - Cotd. AImaxi}

Risultati analitici

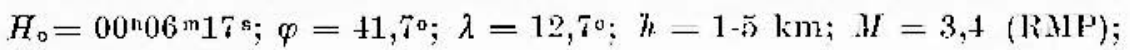
$I_{0}=\mathrm{V}$

\section{Descriztone Alel periodo sismiro}

28-1X, 15 $5^{\mathrm{h}} 4^{\mathrm{m}} 3 \mathrm{~s}^{\mathrm{s}}$, Sentito di III a Colonna, Borgata Finorehio. Seguono topo questa altre tre scosse, che risulteramo poi premonitrici della suceessiva.

29-IX, $00^{\mathrm{h}} 0 \mathrm{6}^{\mathrm{m}} 1 \mathrm{~T}^{5}$. Scossa principale, sentita ti $\mathrm{V}$ a Colonua, di IV alla Borgata Finocchio, di III a Frascati e Grollaformata. Isa profonclità ipocentrale risulta lra 1 e $5 \mathrm{~km}$, la marniturlo è di 3,4 . Seguono nello stesso griono tra le $00^{\mathrm{n}} 33^{\mathrm{m}}$ e le $19^{\mathrm{b}} 1 \mathrm{l}^{\mathrm{m}}$, ben 17 repliclec, con le stesse carntteristiche dubla procedente mat piǹ lievi.

30-IX, Altre tre lievi scosse.

$5-\mathrm{X}, \quad 10^{\mathrm{t}} 17 \mathrm{~m}^{\mathrm{s}} \mathrm{s}^{\mathrm{s}}$, debole scossa di magniturlo 3,0 .

$7-\mathrm{X}, \quad 18^{n 16 m 5} 5^{5}$, seossa debolissima.

$13-\mathrm{X}, \quad 01^{\mathrm{l}} 03^{\mathrm{m} 13 \mathrm{~s}}$, debole scossil di maguitudo 3,0 . Seguono tra le $10^{\mathrm{m}} 17^{\mathrm{m}}$ e le $21 \mathrm{~b} 16^{\mathrm{m}}, 3$ scosse con masniturlo infuriore alla precedente ed altre 13 di debolissima intensita.

14-X, tra le $01^{\mathrm{n}} 59^{\mathrm{m}}$ e le $04^{\mathrm{h} 08^{\mathrm{m}}}, 4$ lievi soosse.

18-X, $03^{n} 46^{\mathrm{m}} \mathrm{5}^{\mathrm{2}} \mathrm{s}$, avvertita di III-IV lumgo la via Casilina. Con intensil à minore si è manifestata as. Cesareo e Grottaferata. Tei gromi successivi seguono 4 repliche.

24-X, avvertito di 111 a Colomma.

(*) Bollettino I.X.t. - Settembre-0ttobre-Soxembre-Dicembre 1970.

Bollettimn I.S.C.(t.S. - 1970).

Bollettino B.(. 1.S. - 1970).

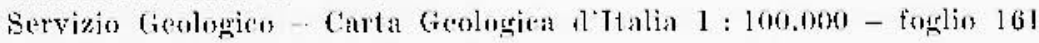
"Isernia" - foglio 160 "Cassinon. 
18-XII, $05^{m} 25^{m}{ }^{m} 0 s$, sentito di III a Laghetto, il giomo precedente due sctossee.

22-XII, 12n40 ${ }^{m} 54^{s}$, scossa rebole, nei giorni precenlenti si sono avute t.re scosse.

In base agli azimut misumati su sismogrammi, el ai tempi di arrivo delle varie onde si è posto l'epicentro ad una slistanza di $7 \mathrm{~km}$ cirea dall'osservatorio, e eioè in prossimità del cono vulcanieo ali M. Falcone.

\section{Considerazioni geologichc}

L'atiule sisma ha interessato larea posta a Ford dell'apparato vulcanico dei Colli Albani (11). Questa zona tettonicamente i interessata da fratture con direzione NE-SWV e NW-SE ddirezioni appenninica er antiappenuinica). Generalmente alloncontro sli tali fratture, hanno origine i dabocelli lavier come quello di Laghet,o, o come i eumuli cupuliformi di M. Falcone, che sono stati interessati dugli ipocentri dell'attuale periodo sismico.

Le isomomale regionali mettono in evidenza lungo il versante $\mathrm{NE}$ del sistema vuleanico, importanti frature parallele all'asse del vuleano stesso.

Il perioto sismico ha interessato il borto settentrionale dell'orlo di caklera precerlente al tufo di Villa Semi cioè al limite della zona «li spmofonclamento de]l'applatito vulanico laziale (*).

10) - 31 Diembre - Jagunia OChDLNThLE

$H_{o}=22^{\mathrm{n} 0} 04^{\mathrm{m}} 46^{\mathrm{s}} ; \varphi^{-}=44^{\circ} 09^{\prime} ; \lambda=8{ }^{\circ 1} 7^{\prime} ; h=9 \mathrm{~km} ; M=4,3 ; I_{0}=\mathrm{VI}$

Altre determinazioni epicentrali

(U.S.C.G.S.) $\Pi_{0}=22^{\mathrm{n}} 04^{\mathrm{m}} 47^{\mathrm{s}, 9 ;}, \varphi=44,4^{\circ} ; \lambda-8, \tilde{\mathrm{j}}^{\mathrm{o}} ; \quad H=4,1 ; h-\mathrm{N}$

(B.C.I.S.) $\quad H_{0}=22^{\circ} 04 \mathrm{~m}^{\mathrm{m}} 46^{\mathrm{s}} ; \quad \phi=14,2^{\circ} ; \lambda=8,2^{\circ}$.

\section{Notizie maerosismiche}

La zona rolpita è la Liguria Occistentale, entroterra e riviera di ponente. Il sisma si ì manifestato con 6 scosse di II grado, 2 di IV grado, 1 di $Y$ grarto, 1 di VI.

(*) Bollettino 1.X.C. . Settembre-Ottobre-Novenbre-Diesmbre 1970 . Scevizio Geologieo - Carta Geologiea dlialia ] : 100.000 - foglio 150

"Romat 1967 - Carta Gravinetrica dlitalia $1: 100.000$ - fogglio 150

"Rollia n 1967. 


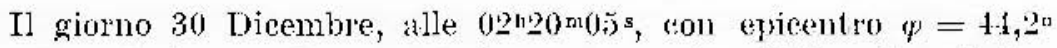
$\lambda=8,3^{\circ}$ (calcolato graficamente), è stato avvertito di Y grado a Calice Jigure.

I dati forniti sono $\mathrm{i}$ seguenti:

(U.S.C.G.S.) $H_{n}=02^{n} 20^{m} 05,8^{\circ} ; p=44,4^{\circ} ; \lambda=8,2^{\circ} ; h=\mathrm{N} ; . M=4,0$

(B.C.I.S.) $\quad H_{\mathrm{o}}=02 \mathrm{~b}^{\circ} 0^{\mathrm{m} 05^{\mathrm{s}}} ; \quad \phi=44,2^{\mathrm{n}} ; \lambda=8,2^{\circ} ; M=4,1$.

Alle 6 scosse si sono avute nel Gemaio 1971 comprese tra il II e IY gralo.

Sono state raccolte le seguenti notizie macrosismiclue:

VI a Pietra Ligure;

$v$ a Calizzano, Lomo, Calice Ligure, Oreo Feglino, Magliolo, Hillesimo, Joggo;

IV a Bagnasco, Spotorno, Osiglia, Xontezemolo, Ceriale, Borghet to \$. Spirito, Caprauna, Giusvalla;

III a Lerna, Rossiglione, Savona, Andora, Paroli Jigure, Savignone, Voltaggio, Gorzegno, Paroldo, Alassio, Monbarcaro, Rocea Grimalda, Tiglieto;

II a Chiavari, Varaze, Genova, Casaleggio, Boiro, Campomorone, Mele, prunetto.

Ja un esame di dettaglio delle isosiste (Fig. I8) si nota clie qualle fli $V$ e VI grado sono lutte contenute nella zona di affiomamento dalla serie Brianzonese. Le altre isosiste IV-III-II, lendono a restringersi verso SW. Probabilmunte questo è dovuto alla presenza delle forma-

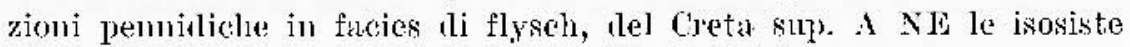

$$
\text { TAB ELSA } 9
$$

\begin{tabular}{|c|c|c|}
\hline Direzione dall epicent ro & $\begin{array}{l}\text { Dislanza } \\
\text { kul }\end{array}$ & $\begin{array}{l}\text { Valore tel coctficientes } \\
\text { di at in enuzione }\end{array}$ \\
\hline $\begin{array}{l}\text { Perpendicolarmente alla linea spar- } \\
\text { fiactue delle } A \text { pi Ligniti. }\end{array}$ & 30) & $0,0 \leq 423 \div 0,0033$ \\
\hline Verso N.T. a $\quad$ S.W. . . & 8 & $(1,1) 6(17001+\ldots 10,(100) 2$ \\
\hline Versu N.E. e s.W. . . . . . & 23 & $0,103485 \pm 10,003320$ \\
\hline 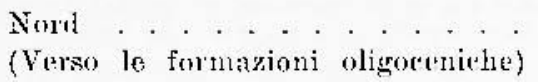 & 34 & 0,0106833 \\
\hline $\begin{array}{l}\text { Nord } \\
\text { (Verso le formazioni oligroceniche) }\end{array}$ & 15 & 0,010999 \\
\hline
\end{tabular}




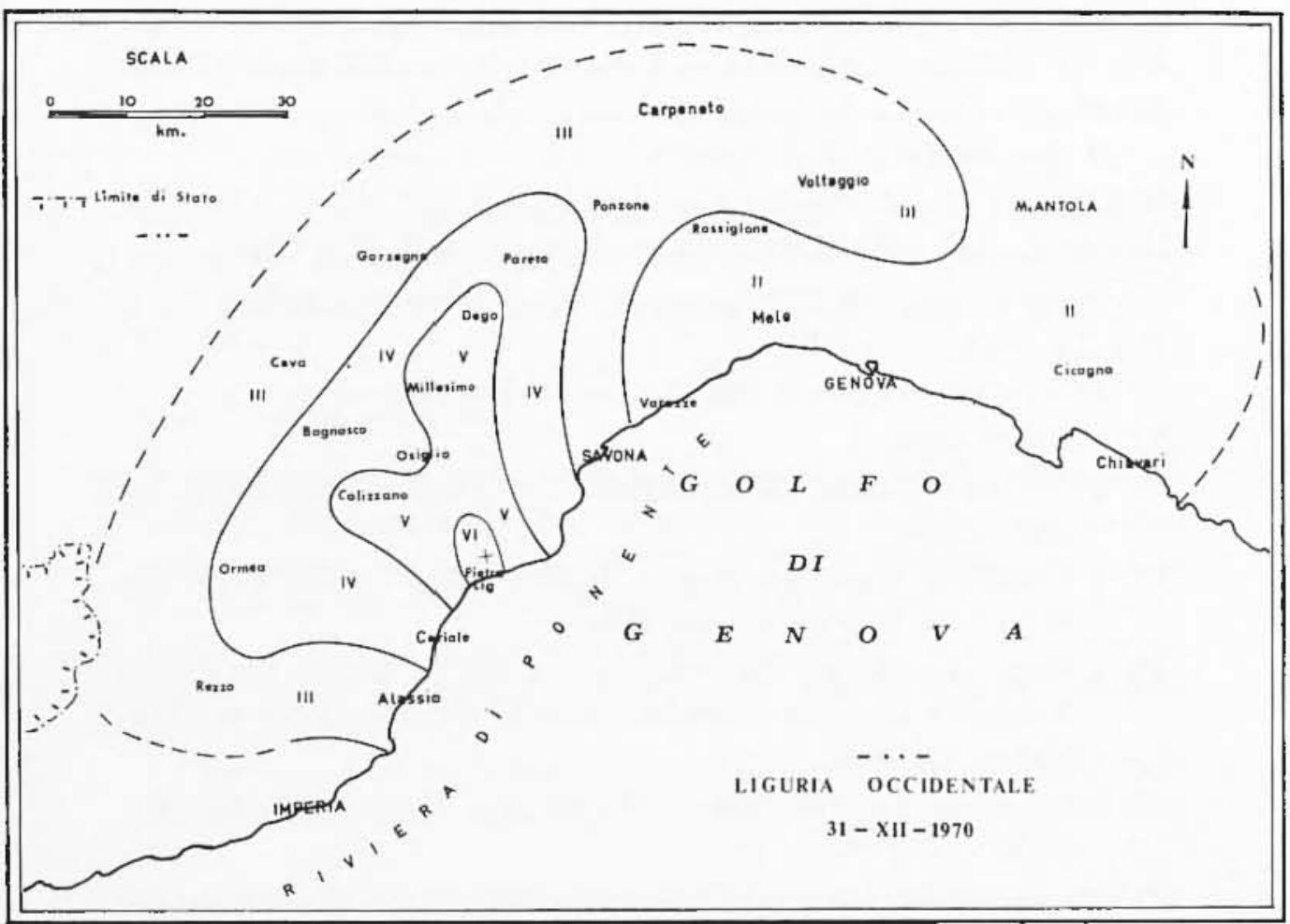

Fig. 18

raggiungono le roce ultrubasiche di Voltri (pietse verdi) e si propagano oltre la linea Sestri-Toltaggio.

I valori del coefficiente d'attenuaione sell'energia macrsismiat in superfieie, sono riportati in 'lab. 9.

\section{Considerazioni geologiche}

La zoma colpita sal sisma e quella in eni affiomono le radiei del Paleonpennino Tirrenico, che a al contine tra Alpi Hartime ed Appennino Ligure. La zona 2 interessata a Oceislente dal kovascorimento al ceriale che si riallaceia piu a ford alla linea di sovraseorrimento delle Pennili sulle Elvet idi. Ad Oriente, invece, è limitata dalle formazioni slella "zonar piemontese ".

I rapporti tettonici si rivelano alquanto complicati. Le principali linee strutturali, sovrascorrimenti, hamo direzione di-W mentre le principali faglie si orientano verko NXW-SSE. 
Lungo la direzione SW-NE si sviluppa un altro sistema di faglie, il quale presenta caratteri meno marati dei sistemi precedenti ed un'area di distribuzione miuore. Lungo faglio di questo ultimo tipo si sono localizzati gli epicentri dellattuale periodo sismico. Prevalentemente $\dot{c}$ stato interessato il sistema di faglie che vamno da Spotorno ad Oreo Feglino, e da questi, al torrente Nimbolto $\left(^{*}\right)$.

(*) Bollettino I.N.G. - Dicembre 1970.

Bollettino U.S.C.G.S. - 1970 .

Bollettino B.C.I.S. - 1970.

Servizio Geologieo - Carta Cieologiea al'Italia 1 : 100.000 - foglio 92.93 "Albenga-Sarona " 1970 - foglio 81 "Cera" 1970 - e relative note illustrative.

\section{BIBLIOGRAFIA}

(1) CaLor 1'., I9f1. - Determinazione delle coordinate epicentrali di un terremoto ad origine ricina con i tempi delle onde longitadinali e trasuersali diretie. "Ricerea beientifica n, XIX, 4.

(2) Calor P., Pekosaci F., 1948, - Il terremolo del Turkesian del : S Vovembre 1910. "Annali di Gicofisica", I.

(3) Canor P., 1934. - Calcolo delle mofondita ipocentrali in funzone della

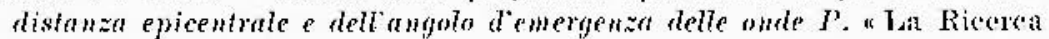
Secientifica", V, 3-4.

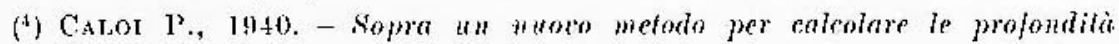
ipecenbali, "La Ricerea sicintifica " XI, 1.

(5) CA101 P., 1957. - C'atratleristiche della mosta terrestre dalle Alpi agli Appennini. "Annali di Geofisica n, $\mathbf{X}$.

(6) C.N.R., 1972. - Relariome sui rilieri effelluati nell area flegrea nel 1970. 1971. Quademi della "Riecrea sicientifiea n 83.

(7) Commission scismologique Europrenne - XII Assemblec ficherale. Eu. J. IJ. Vin Gils - 1971.

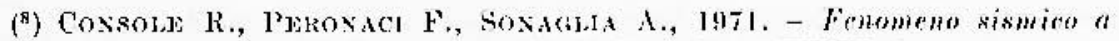
Wigurno Wonle Lango. a Anmali di Geofision n, XXIV, I.

(9) DaLlax Narot L., ELTEK l', Nard R., 1971, Conmiderazioni sullareo dell tppennino Seltewtrionale e sulla "Linea" incona-duzio. "Boll. Soc. (ieol. It. ", XC.

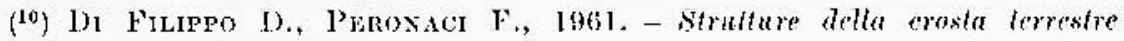
nelle Prealpi Lombardo. Tenete quale risulta... ece. "Ammali di Geotisica a XIV. 
(ii) Porsaseri M., Scherilo A., Vextrichia [', 1963. - La regione Tuleamien dei Golli Albani. C.N.R., Roma.

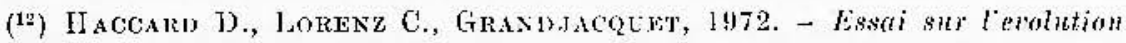
Tectogenctique de la tirison Alpes-A pemmins (De la Ligurie di la C'atobrie). "Memor, Soce Geol. It. ", XI, 4.

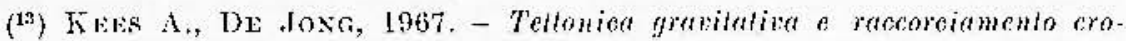
state nette 17 " Weridionti. "Boll. Soc. Geol. It." LXXXVI.

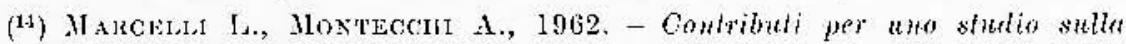
sismicità dell Italits. "Anmali di Geofisiea " XV, 2-3.

(15) Pesta F., 1954. - Ricerble e studi sui fenomeni esulatizo-idrotermali ed it problemo delle "Forze Emdogene" "Anuti di feofisiean, VIII.

(16) PrEsi H, 1967-1968. - Lezioni di geologia regionate. S.T.E.M. MueciModena, I, II, III, IV.

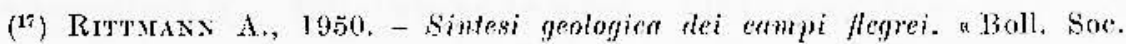
(ieol. It:il. ", LXIX.

(18) Ritrmaxx A., 1967. - I valeani e la foro athitit. Calpyelli Editore.

(19) Takarasi R., 1929 . - 1 graphical determination of porition of the hypoocutre of an earthefutie and the velocity of the popagation of the seismis waves. "Bull. of the Earth", Researcls Institut, Tokyo Imperial University, VI. 


\section{RECENSIONI}

J. Covloms, Sca Floor Sqreading and Conlitental J)ifh (Volume 20 di "Geophysies and Astrophysies Honographs"). Th volume di $\mathrm{x} \div 184 \mathrm{pp}$., con 124 figg. Jiditrice: D. Reitel P'ublishing Company, Dordrecht (Olauda). Nov, 1972, Dfl. 31.

Nel l Cap. l'A. si sofferma sulla geografia sismica, intesa come delimi. tazione delle zone sismiche alla superficie ded trlobo, e sulla batimetria ocea. niea. Nella geografia sismien sono esaminate, in molo particolare, le zone associate alle pieghe del Terziario (fascia del Parifieo, del Meditermene, ece.) e gli archi insulari, nonche le dorsali sommerse con cenni sul mectanismo alliponentro e alla direzione telle tensioni tettoniche. Nel II Cap. si esaminano i risultati delle misure magnetirhe marine, con riferinento esplicito alle anomalie osservate nei pressi degli archi insulari, deile dorsali sommerse e delle fratture trasrersali. Segue un breve capitolo sull'immersione del campo magnetico terrestre e sui fenomeni connessi, mentre il V Cap. è tedicato all'espansione degli ocenini, delinitala fra le dorsali e le fosse oceaniche. La deriva continentale forma argomento ded $V$ (ap. con disenssione di varic ipotesi, compresa la piu recente sulle grandi placelue sferiche rieride ("Rigit Plate IIynothesis"), limitate dalle fascie sismiche, o con la ricostruzione dei bloceli continentali. I Cap. VI e VIT riferiscono rispettivamente sullo stutio della crosta oceanica metiaute la sisnica a rifrazione o la gravimetria esulla corrente di calore attraverso il fondo delloceano. La natura del mantello superiore e le teoric sulla convezione formano argomento idel Cim. VIIT, mentre il capitolo IX si sofferma sul raffronto continenti-oceani consisterauto il fonto tegli orani cone cinghia di trasmissione. L'ultimo capitolo (il $X$ ) riassume i più recenti progressi, con riferimento alle anomalie nuarretiche e al fondo thel mare, alle torsali es alle spatecature, alla tinamicat e termodinamica del moto delle placehe rigirde, ai mari precontinentali, allorogenesi nellambito della teoria delle placehe rígide, eec.

Una sintesi cflicace sopra una materia in fase di transizione, dove insieme a fruthose intuizioni non mancano conclusioni in eui non poeo gioca la fantasia.

P. C. 
*** $x$ - and ficmma - Ray Astronomy (Internativnal Astronomieal Union, Symposium N. 55), a curat di H. Bradt e $R$. Giaceni. Un volume di $\mathrm{x}+323$ p). con numerose figg. nel testo. Editrice: D. Reidel Publishing Company, Dortrecht (Olanta). 1973, Dft. 68.

Riporta gli Atti ale 550 Simposio dell'[AU, tenuto a Aadrid fra l'il e il 13 Magrio 1972 .

Nelle note introduttive, Bruno Rossi rieorda che il simposio cate nel decino aniversario della nascita dell'astronomia per raggi $\mathrm{X}$, che ha gria compiuto sorprendenti risultati, segruita a breve distanza dall'astronomia per raggi Gamma costretta a lavorare fra notevoli difficolti teeniche per la recezione di Russi thbolissimi tli raggri $\gamma$.

TA commicazioni presentate si riferiscono n sorgemti galatlehe (Parte $\mathrm{I}$ ), con particolare riguardo ai raggi $\mathrm{X}$, con simultanee osservazioni ot liche radio e accenui alla polarimetria, la spettroseopia ad olevata rikoluzione slei raggi $\mathrm{X}$ galaltici; ai modelli teoriei per sorgenti eompalle (Parte 11 ), anche con riferimento alle "pulsar "; alle sorgenti extragalattiche con riferimento alle loro proprieti, tratte dalle osservazioni oltiehe, a al loro enntributo alla diffusione di foudo; alla materin interstellare e alla ricezione delle radiazioni $X$ di fondo, comprendenti anche l'assorbimento e la produzione di deboli raggi $\mathrm{X}$ nella Galassia e atromentazioni teoriche sui ragri $\mathrm{X}$,li fonto.

Il volume si conclude con una lunga discussione sull'onergia dei raggri $\mathrm{X}$ sullo sfondo extragalatico difiuso e sull elevata cuergia di sorgenti clistinte.

I. C.

Diddens A. N., P'ilkunn [1., Schlupmane K., Properties and production speotra of elementary particles - Figenschaften und Fraegungsspoltren von. Flementarteilehen. (Volume 6 delia raceolta " Jandolt. Bärnstein * sui dati numerici e le relazioni fumbionali nella scienza e nella Teenica). A eura di H. Schopper. IIn volume di xt +164 ple, con 30 figg. Wditrice: Springer-Verlag, Berlin-IJeillellyerg-New Fork 1972, D) 118.

F, il primo volume della Nuova seric del "T Jandolt. I3örnstein " (6\% dell'intera raccolta) e conticue una eompilazione di dati sulle particelle elementari.

Ja prima parto compilata da P'ilkuln trelp'Tniversita di Karlsune, si riferisee alle proprieti delle particelle, alle costanti al accoppiamento e ai fattori di forma, e si sofferma sulle formule per il decadimento e la risonauza, sulle formule generali per le reazioni di due particelle, sulle tavole delle propricti delle particelle e sulie costanti di decatimento e di aceoppia. mento, sui fattori di forma eletimmagnetici, ece.

Ja seconda parte (rexlatta cla Carlson, Disldens, Giacomelli, Schlüpmann e Schoppery sviluppa le notazioni e relazioni, con particolare riferimento alle sezioni trasversali, alla polarizzazione, alle invarianti relativistiche, alle trasformazioni di Jorentz, ece, nonche le unitid e le costanti.

Ja terza parte infine ha per argomento lat produzione ali particelie nelle interazioni protone-protone ed è compilata da Didlens folel CRRN 
di Ginevral) es da Schlupmann felell Lniversita di Berlino). Contiene, fra l'altro, la sistematiea della proslazione di partieclle, la teuria e le formule empiriche, uno sguardo generale sugli esperimenti of te tabelle di tati sulle sezioni alurto per la produzione si particelle cariche nelle collisioni "pp" come funzione di liverse variahili einematiche.

l'. C.

*** I'roceetlings of the Thind Tmernational Conference on Numerical Irethods in Flaid Ifechamics. Tol. I: Genteral Lectares. Fundamental Numerical Techniques (in "Jeeture Notes in Physies", n. 18). A eura ti IH. Ca. bannes e R. Temam. Un rolume ti vil-186 pp., con 98 figg. EAlitriet Springer-Verlag: Berlin-IIei«lelber-New York 1973, DMl 18.

Custitusee la prima parte dei Renticunti della Ter\%a Conferenza 1nternazionale sui metodi numerici nella Mlecantea dei fuchi, tenutasi a $P_{a}$ rígi (Università VI e XI) dal 3 al 7 Jaglio I 972.

La parte generale contiene una revisione tei metodi per la risoluzione Aclle equazioni di Navier-Stokes (Jotornicyn), una nota sulla Dinamica atmosferiea (Mlorel) e un breve riesane dei motodi per le correnti con urto (Richtmper).

Seguno trentici comunicazioni sulle tecniche numeriche fontamentali (per ma rapida soluzione di problemi con valori al contorno, sull approssimazione delle funzioni a divergenza nulla con il metodo degli elementi finiti, sui metodi alle aliferenze finite per lo stato stazionario nelle equazioni 1i Navier-Stolies, sugli sehemi numerici invarianti di gruppo per le equilzioni della dimamion dei gas, eec.).

13. C.

*** Procedings of the Third International Conjerence on Nimerical Methods in Flaid Mechanics. Fol. II: Prohlems of Fluid Mechanies fin "Lecture Yotes in l'sysies", n. 19). A "ura di H, Cabannes e R. Temam. Lin volume di viI +275 plu. con 176 figf. Editries: Springer-Yerlag BerlinHeidelberg-New York 1973 , DMI 26.

Conticne 35 commienzioni su problemi vari di propagazione, ti correnti, di onde d'urto, di onde a'esplosione, ece. (oscillazioni libere sli wn baciuo in rotazione, calcolo d'un afflusso viseoclastico in una caviti quadrata, studio tlegli efflussi transsonici cun onde d urto, transizioni di turbolenza in corrente convettiva, soluzione numerica dell'efuazione di trasporto vorticoso, strutura di un'onela diurto obliçua rifiessa, caleolo di correnti separate a velocita subsuriche e tramssonithe, soluzioni numeriehe nei problemi tli propagazione per onte d'esplosione, soluzioni mumeriche delle equazioni di Navier-Stokes per eflussi in strati viscosi, problemi di meecanica dei fluidi, non lineari, stazionari in Meteorologia, tece.).

P. C. 